INFORMATION CIRCULAR 32

\title{
DGGS COPY
}

DIRECTORY OF AGGREGATE, ROCK, AND SOIL PRODUCERS IN ALASKA

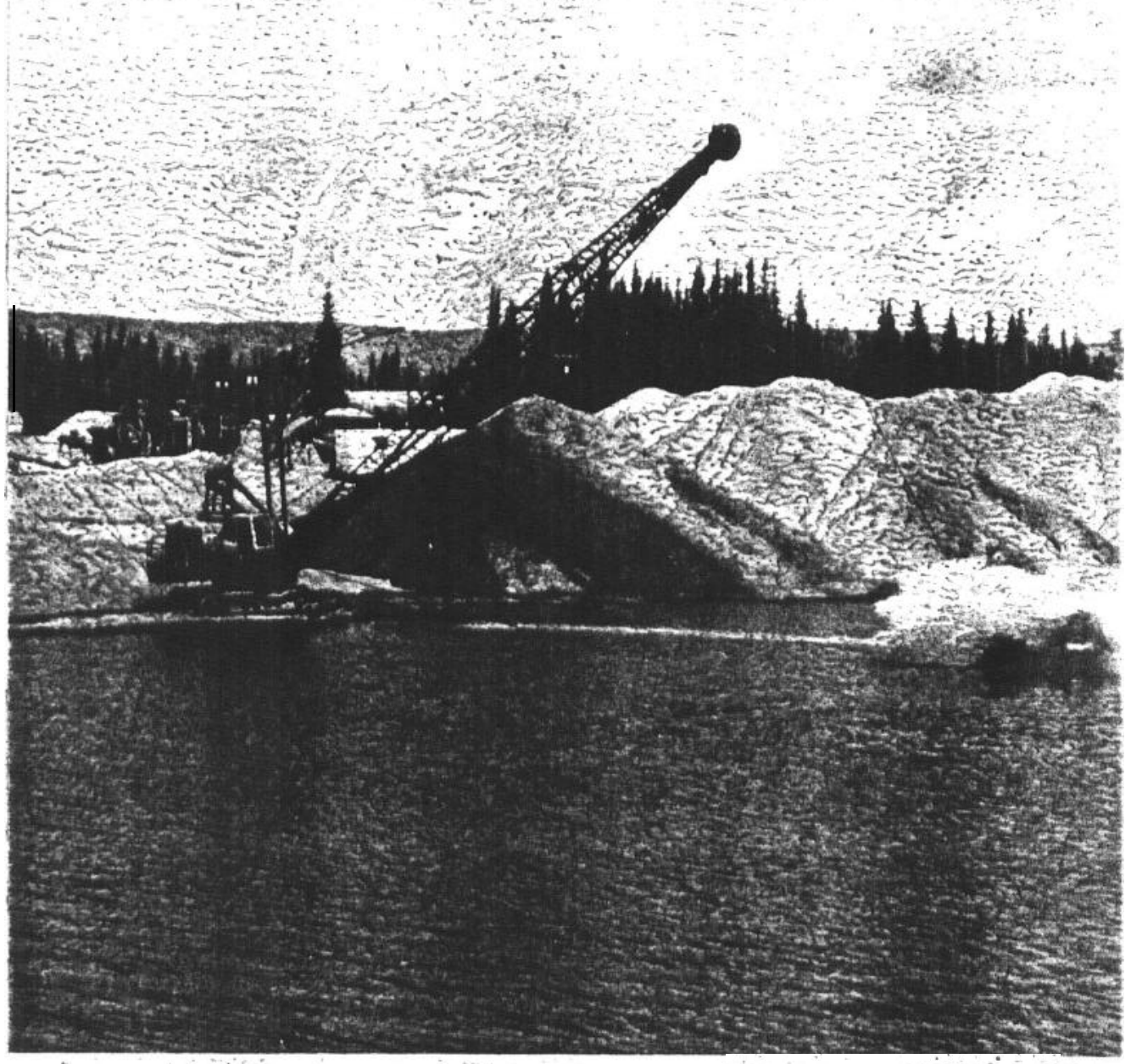

State of Alaska|

DIVISIỎNOF GEOLOGICAL \& I) GEOPHYSICAL SURVEYS

December 19901

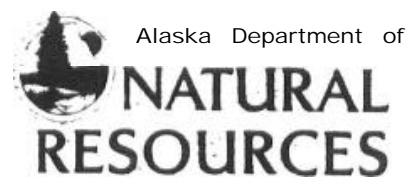




\title{
DIRECTORY OF AGGREGATE, ROCK, AND SOIL PRODUCERS IN ALASKA
}

\section{INFORMATION CIRCULAR 32}

\author{
Published by \\ DIVISION OF GEOLOGICAL \& \\ GEOPHYSICAL SURVEYS
}

\author{
Robert B. Forbes \\ State Geologist
}

Fairbanks, Alaska

1990

Cover: View northeast of dragline with Barbie-yard bucket excavating sandy gravel from Earth Movers, Inc. pit on Hanson Road near Fairbanks, A laska. Photograph taken June 16, 1989. 


\section{STATE OF ALASKA Department of Natural Resources DMSION OF GEOLOGICAL \& GEOPHYSICAL SURVEYS}

According to Alaska Statute 41, the Alaska Division of Geological and Geophysical Surveys is charged with conducting "geological and geophysical surveys to determine the potential of Alaskan land for production of metals, minerals, fuels, and geothermal resources; the locations and supplies of ground water and construction materials; the potential geologic hazards to buildings, roads, bridges, and other installations and structures; and shall conduct such other surveys and investigations as will advance knowledge of the geology of Alaska."

In addition, the Division of Geological and Geophysical Surveys shall collect, record, cvaluatc, and distribute data on the quantity, quality, and location of underground, surface, and coastal water of the state; publish or have published data on the water of the state and require that the results and findings of surveys of water quality, quantity, and location be filed; require that water-well contractors file basic water and aquifer data, including but not limited to well location, estimated elevation, well-driller's logs, pumping tests, flow measurements, and water-quality determinations; accept and spend funds for the purposes of AS 41.08.017 and 41.08.035, and enter into agreements with individuals, public or private agencies, communities, private industry, and state and federal agencies; collect, record, evaluate, archive, and distribute data on seismic events and engineering geology of the state; and identify and inform public officials and industry about potential seismic hazards that might affect development in the state.

Administrative functions are performed under the direction of the State Geologist, who maintains his office in Fairbanks. The locations of DGGS offices are listed below:

-794 University Avenue

(Suite 200)

Fairbanks, Alaska 99709

(907) 474-7147

- 3700 Airport Way

Fairbanks, Alaska $99709-4699$

(907) 451-2760
-400 Willoughby Avenue (3rd floor)

Juneau, Alaska 99801

(907) 465-2533

- 18225 Fish Hatchery Road P.O. Box 772116

Eagle River, Alaska 99577

(907) 696-0070

DGGS publications may be inspected at the following locations. Mail orders should be addressed to the Fairbanks office.

- 3700 Airport Way

Fairbanks, Alaska 997094699

- U.S. Geological Survey

Earth Science Information Center 605 West 4th Avenue, Room G84

Anchorage, Alaska 99501
- 400 Willoughby Avenue (3rd floor)

Juneau，Alaska 99801

*U.S. Geological Survey

Earth Science Information Center 4230 University Drive, Room 101 Anchorage, Alaska 99508 


\section{CONTENTS}

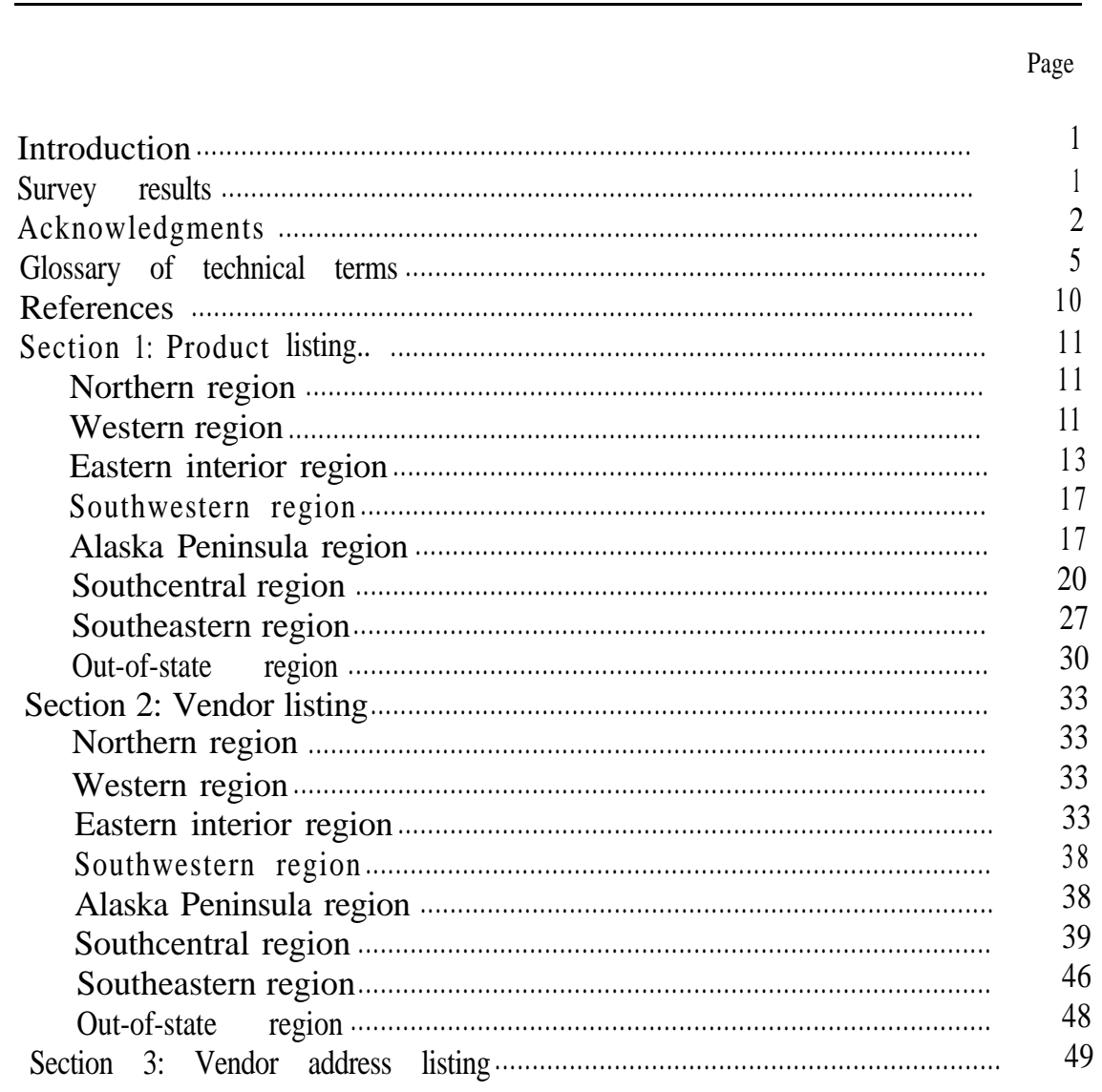

FIGURE

Figure 1. Map showing locations of regional subdivisions of Alaska relative to selected major settlements

\section{TABLE}

Table 1. Regional distribution of vendors providing 29 categories of aggregate, rock, and soil products in Alaska ................................... 
This publication was released by the Division of Geological \& Gcophysical Surveys to provide information of active vendors of aggregate-related products in Alaska. The purpose of this report, required by AS 41.08.017, is to encourage development of Alaska's sand, gravel, rock, and soil resources. Seven hundred copies were printed in Fairbanks, Alaska, at a cost of $\$ 2.40$ per copy. 


\title{
DIRECTORY OF AGGREGATE, ROCK, AND SOIL PRODUCERS IN ALASKA
}

\author{
BY \\ Richard D. Reger and Rodney A. Combellick ${ }^{1}$
}

INTRODUCTION

Since 1981, the Alaska Division of Geological and Geophysical Surveys has published for public use general information on the volumes and values of sand and gravel and other rock materials produced annually in Alaska (Bundtzen and others, 1982, 1984, 1986, 1987, 1988; Eakins and others, 1983, 1985). Each year, we also receive numerous requests for information on who supplies various aggregate, rock, and soil products in our state. The purpose of this directory is to help potential customers locate active vendors of these materials.

\section{SURVEY RESULTS}

For the purposes of this compilation, Alaska is divided into eight regions (fig. 1). A questionnaire was developed to collect basic information on the types of materials being produced in our state by industrial organizations identified during preliminary surveys of business licenses, mining licenses, and telephone directories, and by direct telephone contacts. No formal specifications for materials classes were sent with the questionnaires; products were merely listed. Questionnaires were not sent to major land holders or government entities, even though many sell or produce aggregate, rock, and soil materials. These sources will be verified by a subsequent survey. Of 331 possible vendors initially identified, 103 (31.1 percent) completed and returned questionnaires.

Data from each response were entered into a computerized file for storage, sorting, and retrieval. Based entirely on information received from these active producers, vendors for 29 categories of sand-and-gravel, rock, soil, and related products are identified in each region, as well as instances where no vendors are known (table 1). In cases where we know that certain vendors produce materials that they did not indicate, such as placer metals (byproducts), we have not listed these items.

The greatest selection of products and vendors exists in the eastern interior and southcentral regions. In contrast, no private vendors are headquartered 'Alaska Division of Gcological and Geophysical Surveys, 3700 Airport Way, Fairbanks,
Alaska 99709499. 
in the northern and southwestern regions, where most of the aggregate is produced by petroleum companies, by various Native organizations, or by private organizations that are actually headquartered elsewhere. Responses from 13 companies (12.6 percent) indicate that they serve regions outside their home region, but others undoubtedly do also. Only one vendor outside Alaska responded to our questionnaire.

Material categories for which few (one to three) active vendors have been identified include glass sand, furnace slag, marble, limestone-travertine, lime, gypsum, barite, potting clays, light-weight aggregate, and byproducts. Other vendors undoubtedly offer these products but did not respond to our survey.

The first section of this directory lists vendors providing each of the 29 categories of materials in each region; categories for which no vendor has been identified are also shown. The second section lists aggregate products provided by each vendor in each region. Section three provides a complete alphabetical listing of vendors, including current addresses, key contact personnel, and current telephone numbers. A glossary defines technical terms used in this circular.

Our experience in locating active producers of sand, gravel, rock, and soil indicates that, in this industry, names and addresses of small companies especially seem to change frequently, perhaps reflecting changes in organization or viability. Users of this directory are urged to verify the products currently being offered by each vendor listed and to ensure that these products meet their needs. To provide as accurate information as possible, we plan to update this directory every 2 years.

\section{ACKNOWLEDGMENTS}

This circular would not have been completed without patient, tedious work by Janette M. Heral and Bret E. Davidson, student interns in the DGGS Engineering Geology Section. Milton A. Wiltse and Jeffrey T. Kline of DGGS reviewed an early draft of this directory and provided several very helpful suggestions. We thank Ann-Lillian C. Schell of DGGS for capably handling editing and final production details. 


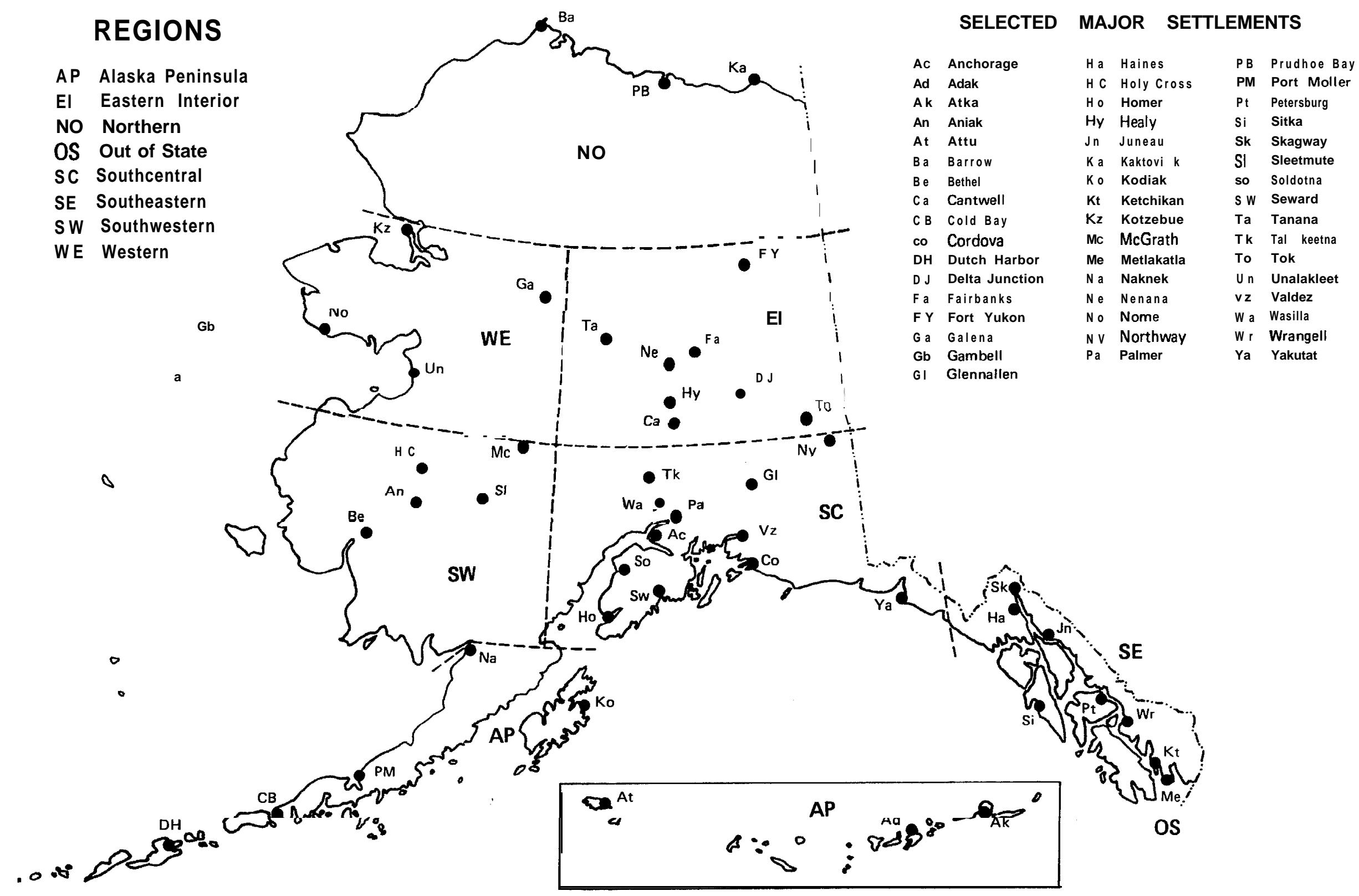

Figure 1. Map showing locations of regional subdivisions of Alaska relative to selected major settlements. 
Table 1. Regional distribution of vendors providing 29 categories of aggregate, rock, and soil products in Alaska (based on 103 responses)

Region

\begin{tabular}{|c|c|c|c|c|c|c|c|c|c|}
\hline \multirow[b]{2}{*}{ Product } & \multicolumn{9}{|c|}{ Region } \\
\hline & Northern & Western & $\begin{array}{l}\text { Eastern } \\
\text { interior }\end{array}$ & $\begin{array}{l}\text { South- } \\
\text { western }\end{array}$ & $\begin{array}{c}\text { Alaska } \\
\text { Peninsula }\end{array}$ & $\begin{array}{l}\text { South- } \\
\text { central }\end{array}$ & $\begin{array}{l}\text { South- } \\
\text { eastern }\end{array}$ & $\begin{array}{l}\text { Out } \\
\text { of } \\
\text { state }\end{array}$ & Total \\
\hline
\end{tabular}

Pit-run gravel

Screened aggregate

Concrete sand-gravel

Ready-mix concrete

Concrete block or similar products

Pavement products

Asphalt sand-gravel

Masonry-mortar sand

Masonry-grout aggregate

Built-up roof aggregate

Filter sand

Blasting sand

Glass sand

Furnace slag

Crushed rock

Dimension stone

Ornamental stone

Riprap-armor-gabion-drain rock

Marble

Limestone-travertine

Lime

Gypsum

Barite

Horticultural peat

Potting clays

Top soil

Light-weight aggregate

Miscellaneous fill materials

Byproducts

Number of respondents

\begin{tabular}{ll}
$\mathbf{0}$ & 2 \\
$\mathbf{0}$ & 0 \\
$\mathbf{0}$ & 3 \\
$\mathbf{0}$ & 1 \\
$\mathbf{0}$ & 0 \\
$\mathbf{0}$ & 0 \\
$\mathbf{0}$ & 0 \\
$\mathbf{0}$ & 0 \\
$\mathbf{0}$ & 0 \\
$\mathbf{0}$ & 0 \\
$\mathbf{0}$ & 1 \\
$\mathbf{0}$ & 0 \\
$\mathbf{0}$ & 0 \\
$\mathbf{0}$ & 0 \\
$\mathbf{0}$ & 1 \\
$\mathbf{0}$ & 1 \\
$\mathbf{0}$ & 0 \\
$\mathbf{0}$ & 1 \\
$\mathbf{0}$ & 0 \\
$\mathbf{0}$ & 0 \\
$\mathbf{0}$ & 0 \\
$\mathbf{0}$ & 0 \\
$\mathbf{0}$ & 0 \\
$\mathbf{0}$ & 0 \\
$\mathbf{0}$ & 0 \\
$\mathbf{0}$ & 2 \\
$\mathbf{0}$ & 0 \\
$\mathbf{0}$ & 1 \\
$\mathbf{0}$ & 1 \\
$\mathbf{0}$ & 3 \\
\hline
\end{tabular}

$\begin{array}{lrrrrr}\mathbf{0} & 1 & 28 & 4 & 1 & 45 \\ \mathbf{0} & \mathbf{0} & 17 & 1 & 1 & 32 \\ \mathbf{0} & 4 & 24 & 5 & 0 & 51 \\ \mathbf{0} & 3 & 9 & 5 & 0 & 24 \\ \mathbf{0} & 0 & 2 & 1 & 0 & 6 \\ \mathbf{0} & 1 & 6 & 2 & 0 & 13 \\ \mathbf{0} & 3 & 13 & 2 & 0 & 23 \\ \mathbf{0} & 2 & 9 & 1 & 0 & 15 \\ \mathbf{0} & 1 & 7 & 1 & 0 & 11 \\ \mathbf{0} & 0 & 2 & 1 & 0 & 5 \\ \mathbf{0} & 2 & 11 & 2 & 1 & 22 \\ \mathbf{0} & 1 & 1 & 2 & 0 & 7 \\ \mathbf{0} & 0 & 0 & 0 & 0 & 1 \\ \mathbf{0} & 0 & 0 & 1 & 0 & 1 \\ \mathbf{0} & 6 & 24 & 7 & 0 & 54 \\ \mathbf{0} & 1 & 6 & 7 & 0 & 20 \\ \mathbf{0} & 2 & 5 & 2 & 0 & 12 \\ \mathbf{0} & 6 & 27 & 11 & 0 & 62 \\ \mathbf{0} & 0 & 1 & 0 & 0 & 1 \\ \mathbf{0} & 0 & 2 & 0 & 0 & 3 \\ \mathbf{0} & 0 & 0 & 0 & 0 & 1 \\ \mathbf{0} & 0 & 0 & 0 & 0 & 1 \\ \mathbf{0} & 0 & 0 & 0 & 0 & 1 \\ \mathbf{0} & 1 & 3 & 0 & 0 & 7 \\ \mathbf{0} & 0 & 1 & 0 & 0 & 1 \\ \mathbf{0} & 3 & 22 & 8 & 0 & 50 \\ \mathbf{0} & 0 & 1 & 0 & 0 & 2 \\ \mathbf{0} & 4 & 23 & 0 & 1 & 47 \\ \mathbf{0} & 0 & 2 & 4 & 0 & 3 \\ \mathbf{0} & 6 & 48 & 16 & 1 & 103\end{array}$




\section{GLOSSARY OF TECHNICAL TERMS}

Aggregate Natural particles, crushed and broken rock, and manmade materials that are graded by size into categories to meet specifications for particular construction uses (Evans, 1978, p. 4).

Armor rock Stone resulting from blasting, cutting, or other methods to obtain pieces heavy enough (generally 1 to 3 tons) to require handling two individual pieces by mechanical means (ASTM, 1988a, Designation D65387). Used to protect beds, banks, shores, and embankments against intense erosion and scour by running water, tidal currents, and wave action.

Asphalt sand andgravel Sized and broken sand and gravel mixed with asphalt in batch plants to form asphalt concrete and road pavements (ASTM, 1988b, Designation D8-87). Dry, clean, fractured particle faces adhere well to bitumen and pack (interlock) efficiently to provide increased concrcte and pavement strength (Evans, 1978, p. 4).

Barite Barium sulfate, which has a specific gravity of 4.3 to 4.6 (Ford, 1958, p. 748).

Blasting sand Abrasive sand, generally composed of angular to subangular, durable (unfractured) quartz grains, used for sandblasting (Evans, 1978, p. 5-6).

Built-up roof aggregate Clean, hard, durable crushed stone and gravel that are free of clay, loam, sand, and other foreign substances and are spread on asphalt roof surfaces (ASTM, 1988b, Designations D1863-86, D1864-81, and D1865-83). Must be opaque to the transmission of light to prevent alteration of underlying roofing materials (ASTM, 1988b, Designation D1866-79).

Byproducts Placer metals, garnet, ilmenite, chromite, rare-earth elements, zircon, cassiterite and other minerals concentrated during the mechanical processing of natural aggregates.

Cobble A rock fragment, usually rounded to subrounded, with an average dimension between 3 and 12 in. (75 and $305 \mathrm{~mm})($ ASTM, 1988a, Designation D653-87).

Concrete A composite construction material made by mixing properly-sized aggregate, a reactive binding medium (Portland cement), and 
water into a slurry (ASTM, 1988b, Designation C125-88). Naturally rounded aggregate particles are desirable because they improve the workability of the mixture when it is poured (Evans, 1978, p. 4).

Concrete blocks Block-shaped structural elements made out of concrete.

Concrete sand and gravel A mixture of appropriately proportioned sand and gravel having a desirably small amount of deleterious substances (weathered feldspars, reactive chert, iron-stone concretions, silt and clay particles, organic matter, friable particles, and detrital coal) to make concrete for specific construction purposes (ASTM, 1988b, Designation C33).

Crushed rock Reasonably clean, tough, durable rock fragments broken by crushing coarse rock fragments or aggregate so that essentially all particle faces are freshly fractured (ASTM, 1988a, Designation D693-84; Langer, 1988, p. 3).

Dimension stone Natural stone that has been selected, trimmed, or cut to specified or indicated shapes or sizes, with or without one or more mechanically dressed surfaces (ASTM, 1988a, Designation C119-87a).

Drain rock Clean, subrounded rock fragments, principally cobble sized, used as fillings in surface and subsurface drainage structures. Informally called leach rock, bull rock, and sewer rock.

Filter sand Generally silica-rich, clean sand that is poorly graded (well sorted) and permeable (Evans, 1978, p. 5-6). Used extensively in water- and sewage-treatment plants and elsewhere for water conditioning and purification.

Furnace slag Nonmetallic, glassy material resulting from solidification of molten silicates and aluminosilicates of calcium and other base elements accumulated simultaneously with metallic iron under atmospheric conditions in a blast furnace (ASTM, 1988b, Designation Cl2588). Granulated for use in concrete and mortars (ASTM, 1988b, Designation C989-88).

Gabion rock Cobble-sized fillings of wire- or plastic-mesh gabion structures used to retard and deflect scouring stream flows as a means of bank protection.

Glass sand Generally silica-rich (quartz) sand used for the manufacture of glass. Must be free of iron oxides and chromium compounds and have a 
minimum of other impurities, including alumina and oxides of calcium and magnesium, and must pass No. 20 U.S. Standard sieve (Evans, 1978, p. 5-6).

Gravel Rounded or subrounded rock particles that pass a 3-in. (76.2-mm) U.S. Standard sieve and are retained on a No. $4(4.75 \mathrm{~mm})$ U.S. Standard sieve (ASTM, 1988a, Designation D653-87).

Gypsum Mineral or rock composed of hydrous calcium sulfate (Pettijohn, 1957, p. 479).

Horticultural peat Fibrous organic plant remains used to condition garden soils.

Light-weight aggregate Low-density, frequently cellular aggregate used to make lightweight concrete. Composed of expanded or sintered clay or shale (haydite) or slate, processed diatomite (diatomaceous earth) or diatomaceous shale, pumice, tuff, scoria, volcanic cinders, processed volcanic glass (perlite), vermiculite, expanded slag, and the products of coal or coke combustion (ASTM, 1988b, Designation C125-88).

Lime Calcium oxide.

Limestone Detrital or chemically precipitated sedimentary rock composed dominantly of calcium carbonate (Pettijohn, 1957, p. 381-382). Where recrystallized, microcrystalline limestone may be capable of acquiring a surface polish and may be sold as limestone or marble (ASTM, 1988a, Designation C119-87a).

Marble Metamorphosed carbonate rock with a distinctive crystalline texture and capable of acquiring a surface polish (ASTM, 1988a, Designation C119-87a).

Masonry-grout aggregate Aggregate composed of natural or manufactured sand or a mixture of sand and coarse particles that passes a 0.5 -in. $(12.5-\mathrm{mm})$ U.S. Standard sieve and is used to make grout for masonry purposes (ASTM, 1988b, Designation C404).

Masonry-mortar sand Natural and manufactured sand used in the preparation of mortar. Artificial mortar sand is produced by crushing stone, gravel, or air-cooled blast-furnace slag to ensure suitable particle shape and size gradation. Deleterious substances include friable and lightweight particles and organic impurities, including clastic coal (ASTM, 1988b, Designation C144-87). 
Miscellaneous fill materials Manmade mixtures of soils and rock products that are not generally classified by texture, although they commonly contain abundant fine material. May contain considerable organic matter and artificial waste materials (ASTM, 1988a, Designation D653-87). Technically not an aggregate but a basic construction material (Evans, 1978, p. 4).

Ornamental stone Natural stone that, because of its distinctive structure, texture, color, and adequate durability, provides an appealing decorator material or art medium. Commonly includes jade, soapstone, serpentinites, agate, marbles, limestones (including travertines), dolomites, granitic rocks, quartzites, and sandstones.

Pavement products Road and airport subbases, bases, and travel surfaces composed of mixtures of asphalt and coarse, crushed aggregate (ASTM, 1988b, Designations D8-87 and D2940-74).

Peat Fibrous organic remains of plants with less than 2.5 percent by dry weight of noncombustible mineral ash that is preserved in marshy and swampy wetlands (ASTM, 1988a, Designation D2607-69).

Pit-run gravel Gravel or sandy gravel that is used without beneficiation after excavation from a natural deposit.

Potting clays Plastic soils composed of rock fragments smaller than $0.002 \mathrm{~mm}$ and platy clay minerals that are used for making pottery ware and figurines.

Ready-mix concrete Concrete that is freshly mixed at the vending site (batch plant) and then transported in a continually agitated, slurry state in mixer trucks to the job site for immediate placement (ASTM, 1988b, Designation C94; Dupras, 1989, p. 10).

Riprap rock Selected hard, angular, quarried stone generally weighing between 25 and 5,400 pounds individually and placed to protect beds, banks, shores, and embankments against wave action, tidal forces, and stream currents (ASTM, 1988a, Designation D653-87; ADOTPF, 1988, p. 305).

Rock Naturally occurring, consolidated materials composed of one or more minerals of the earth's crust (ASTM, 1988a, Designation, C119-87a). Any hard, consolidated materials derived from the earth and usually of relatively small size (Langer, 1988, p. 2). 
Sand Particles of rock that pass a No. $4(4.75-\mathrm{mm})$ U.S. Standard sieve and are retained on a No. $200(75-\mathrm{mm})$ U.S.Standard sieve (ASTM, 1988a, Designation D653-87).

Screened aggregate Aggregate that is separated into different classes of particle size by passing it through combinations of fixed or moving screens with standard-sized openings.

Soil Unconsolidated sediments made up of solid particles of various sizes and compositions and formed by the physical and chemical breakdown of rock (ASTM, 1988a, Designation D653-87; Jumikis, 1962, p. 27). May contain organic matter.

Stone Natural rock material of adequate integrity and quality that it can be quarried and then sawed, cut, split, or otherwise sized, shaped, or finished for specific purposes (ASTM, 1988a, Designation, C119-87a). Natural building stone includes granitic rocks, greenstones, limestones, marbles, massive serpentinite (antigorite), and sandstones,

Top soil Generally fine-grained surface soil, frequently including significant organic matter (ASTM, 1988a, Designation D653-87).

Travertine A variety of layered or banded, porous to dense, crystalline to microcrystalline limestone that is deposited by springs, seeps, or running water (ASTM, 1988a, Designation C119-87a; Pettijohn, 1957, p. 409). 
Alaska Department of Transportation and Public Facilities, 1988, Standard specifications for highway construction, $601 \mathrm{p}$.

American Society for Testing and Materials, 1988a, 1988 Annual book of ASTM standards, v. 04.08 (soil and rock, building stones, geotextiles): Philadelphia, $951 \mathrm{p}$.

1988b, 1988 Annual book of ASTM standards, v. 04.02 (concrete and aggregates): Philadelphia, $749 \mathrm{p}$.

Bundtzen, T.K., Eakins, G.R., and Conwell, C.N., 1982, Review of Alaska's mineral resources, 1981-82: Alaska Division of Geological and Geophysical Surveys report, $145 \mathrm{p}$.

Bundtzen, T.K., Eakins, G.R., Clough, J.G., Lueck, L.L., Green, C.B., Robinson, M.S., and Coleman, D.A., 1984, Alaska's mineral industry, 1983: Alaska Division of Geological and Geophysical Surveys Special Report 33, $56 \mathrm{p}$.

Bundtzen, T.K., Eakins, G.R., Green, C.B., and Lueck, L.L., 1986, Alaska's mineral industry, 1985: Alaska Division of Geological and Geophysical Surveys Special Report 39, 68 p.

Bundtzen, T.K., Green, C.B., Deagen, James, and Daniels, C.L., 1987, Alaska's mineral industry, 1986: Alaska Division of Geological and Geophysical Surveys Special Report 40,68 p.

Bundtzen, T.K., Green, C.B., Peterson, R.J., and Seward, A.F., 1988, Alaska's mineral industry, 1987: Alaska Division of Geological and Geophysical Surveys Special Report 41,69 p.

Dupras, Don, 1989, The grandeur of concrete: California Geology, v. 42, no. 1, p. 3-11.

Eakins, G.R., Bundtzen, T.K., Robinson, MS., Clough, J.G., Green, C.B., Clautice, K.H., and Albanese, M.A., 1983, Alaska's mineral industry, 1982: Alaska Division of Geological and Geophysical Surveys Special Report 31, $63 \mathrm{p}$.

Eakins, G.R., Bundtzen, T.K., Lueck, L.L., Green, C.B., Gallagher, J.L., and Robinson, M.S., 1985, Alaska's mineral industry, 1984: Alaska Division of Geological and Geophysical Surveys Special Report 38, 57 p.

Evans, J.R., 1978, Sand and gravel: U.S. Bureau of Mines Mineral Commodity Profile MCP-23, $22 \mathrm{p}$.

Ford, W.E., 1958, Dana's textbook of mineralogy: New York, John Wiley and Sons, $851 \mathrm{p}$.

Jumikis, A.R., 1962, Soil mechanics: Princeton, D. Van Nostrand Company, $791 \mathrm{p}$.

Langer, W.H., 1988, Natural aggregates of the conterminous United States: U.S. Geological Survey Bulletin 1594, 33 p.

Pettijohn, F.J., 1957, Sedimentary rocks: New York, Harper and Row, 718 p. 


\section{SECTION 1 \\ PRODUCT LISTING}

\section{Northern Region}

No respondents.

\section{Western Region}

Pi t-run Gravel

ALASKA CAB GARAGE

RS CONSTRUCTION

Nome, A K

Kotzebue, AK

Screened Aggregate

No respondents.

Concrete Gravel and Sand

ALASKA CAB GARAGE

CRAWFORD WALSH CONSTRUCTION CO.

RS CONSTRUCTION

Nome, A K

Nome, AK

Kotzebue, AK

Ready-mix Concrete

CRAWFORD WALSH CONSTRUCTION CO.

Nome, AK

Concrete Blocks and Similar Products

No respondents.

Pavement Products

No respondents.

Asphalt Gravel and Sand

No respondents.

Masonry-mortar Sand

No respondents.

Masonry-grout Aggregate

No respondents.

Built-up Roof Aggregate

No respondents.

Filter Sand

CRAWFORD WALSH CONSTRUCTION CO,

Nome, AK

\section{Blasting Sand}

No respondents. 
INFORMATION CIRCULAR 32

\section{Glass Sand}

No respondents.

Furnace Slag

No respondents.

Crushed Rock

ALASKA CAB GARAGE Some, AK

Dimension Stone

ALASKA CAB GARAGE

Nome, AK

\section{Ornamental Stone}

No respondents.

Riprap, Armor, Gabion, or Drain Rock

ALASKA CAB GARAGE

Nome, AK

\section{Marble \\ No respondents.}

Limestone or Travertine

No respondents.

Lime

h'o respondents.

\section{Gypsum}

No respondents,

Barite

No respondents.

\section{Horticultural Peat}

No respondents.

\section{Potting Clays}

No respondents.

\section{Top Soil}

ALASKA CAB GARAGE

Nome, AK

RS CONSTRUCTION

Kotzebue, AK

\section{Light-weight Aggregate}

No respondents.

Miscellaneous Fill Materials 


\section{Byproducts}

CRAWFORD WALSH CONSTRUCTION CO.

Nome, AK

\section{Eastern Interior Region}

\author{
Pit-run Gravel \\ EARTII PRODUCTS \\ IIOWARD ENTERPRISES \\ KNIK CONSTRUCTION CO./LYNDEN TRANSPORT \\ METRO COMPANY \\ NISTLER ENTERPRISES \\ ROLLING STONE GRAVEL \& EXCAVATION \\ SWENSON TRUCKING \& EXCAVATING \\ T \& B GRAVEL \\ WRIGHTS AURORA CONSTRUCTION
}

\section{Screened Aggregate}

A.C.E. GENERAL CONTRACTORS, INC.

ARCTIC REDI-MIX, INC.

EARTH MOVERS OF FAIRBANKS, INC

EVANS INDUSTRIES, INC.

FAIRBANKS PUMPING \& 'I-HAWING

FAIRBANKS SAND \& GRAVEL, INC

GREAT NORTIIWEST, INC.

II. \& II. CONTRACTORS, IXC

MCPEAK ALASKA REDI-MIX, INC.

NISTLER ENTERPRISES

SWENSON TRUCKING \& EXCAVATING

UNIVERSITY REDI-MIX, INC.

YUTAN CONSTRUCTION CO.

\section{Concrete Gravel and Sand}

ARCIIC REDI-MIX, INC

EVANS INDUSTRIES, INC

FAIRBANKS PUMPING \& TIIAWING

FAIRBANKS SAND \& GRAVEL, INC.

GREAT NORTHWEST, INC.

II. \&II. CONTRACTORS, INC.

LAKLOEY, INC

MCPEAK ALASKA REDI-MIX, INC.

METRO COMPANY

NISTLER ENTERPRISES

NUGGET CONSTRUCTION, ISC.

REDMAN CONSTRUCTION

ROLLING STONE GRAVEL \& EXCAVATION

SALCHA SAND \& GRAVEL

SWENSON TRUCKING \& EXCAVATING
North Pole, AK

North Pole, AK

Fairbanks, AK

Fairbanks, AK

Delta Junction, AK

Salcha, AK

North Pole, AK

Nenana, AK

Fairbanks, AK

Fairbanks, AK Fairbanks, AK

Fairbanks, AK

Healy, AK

North Pole, AK

Fairbanks, AK

Fairbanks, AK

Fairbanks, AK

Fairbanks, AK

Delta Junction, AK

North Pole, AK

Fairbanks, AK

Fairbanks, AK

Fairbanks, AK

I Iealy, AK

North Pole, AK

Fairbanks, AK

Fairbanks, AK

Fairbanks, AK

North Pole, AK

Fairbanks, AK

Fairbanks, AK

Delta Junction, AK

Tok, AK

Nenana, AK

Salcha, AK

North Pole, AK

North Pole, AK 
Ready-mix Concrete

ARCTIC REDI-MIX, INC.

EVANS INDUSTRIES, INC.

FAIRBANKS SAND \& GRAVEL, INC.

II. \& II. CONTRACTORS, INC.

MCI'EAK ALASKA REDI-MIX, INC.

UNIVERSITY REDI-MIX, INC.

\section{Concrete Blocks and Similar Products}

FAIRBANKS SAND \& GRAVEL, INC.

II. \& H. CONTRACTORS, INC.

UNIVERSITY REDI-MIX. INC.

\section{Pavement Products}

EARTH MOVERS OF FAIRBANKS, INC.

FAIRBANKS PUMPING \& THAWING

FAIRBANKS SAND \& GRAVEL, INC.

SALCHA SAND \& GRAVEL

Fairbanks, AK

I Iealy, AK

Fairbanks, AK

Fairbanks, AK

Fairbanks, AK

Fairbanks, AK

Fairbanks, AK

Fairbanks, AK

Fairbanks, AK

Asphalt Gravel and Sand

EARTH MOVERS OF FAIRBANKS, INC.

GREAT NORTHWEST, ISC.

H. \& H. CONTRACTORS, ISC.

SWENSON TRUCKING \& EXCAVATISG

YUTAN CONSTRUCTION CO.

Masonry-mortar Sand

FAIRBANKS SAND \& GRAVEL, INC.

II. \& H. CONTRACTORS, INC.

UNIVERSITY REDI-MIX, INC.

Masonry-grout Aggregate

FAIRBANKS SAND \& GRAVEL, INC.

H. \& H. CONTRACTORS, INC.

Built-up Roof Aggregate

FAIRBANKS SAND \& GRAVEL, INC. YUTAN CONSTRUCTION CO.

\section{Filter Sand}

A.C.E. GENERAL CONTRACTORS, INC. FAIRBANKS SAND \& GRAVEL, INC.

H. \& H. CONTRACTORS, INC.

MCPEAK ALASKA REDI-MIX, INC.

SWENSON TRUCKING \& EXCAVATING

\section{Blasting Sand}

FAIRBANKS SAND \& GRAVEL, INC.

MCPEAK ALASKA REDI-MIX, INC.

YUTAN CONSTRUCTION CO.

Fairbanks, AK

North Pole, AK

Fairbanks, AK

North Pole, AK

Fairbanks, AK

Fairbanks, AK

Fairbanks, AK

North Pole, AK

Fairbanks, AK

Fairbanks, AK

Fairbanks, AK

Fairbanks, AK

Fairbanks, AK

Fairbanks, AK

Fairbanks, AK

Fairbanks, AK

Fairbanks, AK Fairbanks, AK Fairbanks, AK Fairbanks, AK

North Pole, AK

Fairbanks, AK Fairbanks, AK Fairbanks, AK 


\section{Glass Sand}

\section{Furnace Slag}

No respondents.

\section{Crushed Rock}

A.C.E. GENERAL CONTRACTORS, ISC. ARCTIC REDI-MIX, INC.

DICKSON ENTERPRISES, INC.

EARTH MOVERS OF FAIRBANKS, INC.

EVANS INDUSTRIES, INC.

FAIRBANKS PUMPING \& THAWING

FAIRBANKS SAND \& GRAVEL, INC.

I l. \& H. CONTRACTORS, INC.

JOHNSON EXCAVATING

KURT'S CONSTRUCTION

LAKLOEY, INC.

NISTLER ENTERPRISES

NUGGET CONSTRUCTION, INC.

SALCHA SAND \& GRAVEL

SWENSON TRUCKING \& EXCAVATING

Y UTAN CONSTRUCTION CO.

Fairbanks, AK

Fairbanks, AK

Fairbanks AK

Fairbanks: AK

Ilealy, AK

North Pole, AK

Fairbanks, AK

Fairbanks, AK

North Pole, AK

Delta Junction, AK

North Pole, AK

Delta Junction, AK

Tok, AK

North Pole, AK

North Pole, AK

Fairbanks, AK

\section{Dimension Stone}

DICKSON ENTERPRISES, INC.

EARTII MOVERS OF FAIRBANKS, INC.

JOHNSON EXCAVATING

KURT'S CONSTRUCTION

SALCHA SAND \& GRAVEL

Fairbanks, AK

Fairbanks, AK

North Pole, AK

Delta Junction, AK

North Pole. AK

\section{Ornamental Stone}

A.C.E. GENERAL CONTRACTORS, INC. FAIRBANKS SAND \& GRAVEL, INC. GREAT NORTHWEST, INC.

Fairbanks, AK

Fairbanks, AK

Fairbanks, AK

\section{Riprap, Armor, Gabion, or Drain Rock}

A.C.E. GENERAL CONTRACTORS, INC. ALASKA LIME COMPANY

DICKSON ENTERPRISES, INC.

FAIRBANKS PUMPING \&THAWING

FAIRBANKS SAND \& GRAVEL, INC.

II. \& II. CONTRACTORS, INC.

JOHNSON EXCAVATING

KURT'S CONSTRUCTION

LAKLOEY, INC.

MCPEAK ALASKA REDI-MIX, INC.

NISTLER ENTERPRISES

NUGGET CONSTRUCTION. INC.

Fairbanks, AK

Cantwell, AK

Fairbanks, AK

North Pole, AK

Fairbanks, AK

Fairbanks, AK

North Pole, AK

Delta Junction, AK

North Pole, AK

Fairbanks, AK

Delta Junction, AK

Tok. AK 
REDMAN CONSTRUCTION

ROLLING STONE GRAVEL \& EXCAVATION

SALCILA SAND \& GRAVEL

$\mathrm{T} \&$ B GRAVEL

YUTAN CONSTRUCTION CO.

\section{Marble}

No respondents.

Limestone or Travertine ALASKA LIME COMPANY

Lime

FAIRBANKS SAND \& GRAVEL, INC.

\section{Gypsum}

FAIRBANKS SAND \& GRAVEL, INC.

\section{Barite}

FAIRBANKS SAND \& GRAVEL, INC.

\section{IIorticultural Peat}

GREAT NORTHWEST, INC.

II. \& H. CONTRACTORS, INC.

WRIGI ITS AURORA CONSTRUCTION

\section{Potting Clays}

No respondents.

\section{Top Soil}

DICKSON ENTERPRISES, INC.

EARTH PRODUCTS

EXCLUSIVE LANDSCAPING \& EXCAVATING, INC.

FAIRBANKS PUMPING \& THAWING

GREAT NORTHWEST, INC.

HOWARD ENTERPRISES

JOHNSON EXCAVATING

NISTLER ENTERPRISES

NUGGET CONSTRUCTION, INC.

REDMAN CONSTRUCTION

ROLLING STONE GRAVEL \& EXCAVATION

SWENSON TRUCKING \& EXCAVATING

T \& B GRAVEL

WRIGHTS AURORA CONSTRUCTION

YUTAN CONSTRUCTION CO.

\section{Light-weight Aggregate}

H. \& H, CONTRACTORS, INC.
Nenana, AK

Salcha, AK

North Pole, AK

Nenana, AK

Fairbanks, AK

Cantwell, AK

Fairbanks, AK

Fairbanks, AK

Fairbanks, AK

Fairbanks, AK

Fairbanks, AK

Fairbanks, AK

Fairbanks, AK

North Pole, AK

Fairbanks, AK

North Pole, AK

Fairbanks, AK

North Pole, AK

North Pole, AK

Delta Junction, Ak

Tok, AK

Nenana, AK

Salcha, AK

North Pole, AK

Nenana, AK

Fairbanks, AK

Fairbanks, AK

Fairbanks, AK 
Miscellaneous Fill Materials

DICKSON ENTERPRISES, INC.

EARTH PRODUCTS

FAIRBANKS PUMPING \&THAWING

FAIRBANKS SAND \& GRAVEL, INC.

GREAT NORTHWEST, INC.

II. \& II. CONTRACTORS, INC.

JOIINSON EXCAVATING

KURT'S CONSTRUCTION

LAKLOEY, INC.

METRO COMPANY

NISTLER ENTERPRISES

NUGGET CONSTRUCTION, INC.

REDMAN CONSTRUCTION

ROLLING STONE GRAVEL \& EXCAVATION

SWENSON TRUCKING \& EXCAVATING

$\mathrm{T}$ \& B GRAVEL

WRIGHTS AURORA CONSTRUCTION

YUTAN CONSTRUCTION CO.

\section{Byproducts}

No respondents.
Fairbanks, AK North Pole, AK North Pole, AK Fairbanks, AK Fairbanks, AK Fairbanks, AK North Pole, AK Delta Junction, AK North Pole, AK

Fairbanks, AK Delta Junction, AK Tok, AK

Nenana, AK

Salcha, AK

North Pole, AK

Nenana, AK

Fairbanks, AK

Fairbanks, AK

\section{Southwestern Region}

No respondents.

\section{Alaska Peninsula Region}

\section{Pit-run Gravel}

LLOYD MOTHERSHEAD

Dutch Harbor, AK

\section{Screened Aggregate}

No respondents.

\section{Concrete Gravel and Sand}

A-K CONSTRUCTION CO.

BRECHAN ENTERPRISES, INC.

LLOYD MOTHERSHEAD

MOORCROFT CONSTRUCTION CO.

Kodiak, AK

Kodiak, AK

Dutch Harbor, AK

King Salmon, AK

Kodiak, AK

Dutch Harbor, AK

King Salmon, AK 
Concrete Blocks and Similar Products

No respondents.

Pavement Products

BRECHAN ENTERPRISES, INC.

Kodiak. AK

\section{Asphalt Gravel and Sand A-K CONSTRUCIION CO. BRECHAN ENTERPRISES, INC. \\ MOORCROFT CONSTRUCTION CO. \\ Masonry-mortar Sand BRECHAN ENTERPRISES, INC. \\ MOORCROFT CONSTRUCTION CO.}

Kodiak, AK

King Salmon, AK

Masonry-grout Aggregate

MOORCROFT CONSTRUCTION CO.

King Salmon, AK

\section{Built-up Roof Aggregate}

No respondents.

\section{Filter Sand}

BRECHAN ENTERPRISES, INC.

MOORCROFT CONSTRUCTION CO.

Kodiak, AK

King Salmon, AK

\section{Blasting Sand}

BRECHAN ENTERPRISES, INC.

Kodiak, AK

\section{Glass Sand}

No respondents.

\section{Furnace Slag}

No respondents.

\section{Crushed Rock}

\section{A-K CONSTRUCTION CO.}

ANDERSON CONSTRUCTION

BRECHAN ENTERPRISES, INC.

F/W MATERIALS

LLOYD MOTHERSHEAD

MOORCROFT CONSTRUCTION CO.

Kodiak, AK

Kodiak, AK

Kodiak, AK

Kodiak, AK

Dutch Harbor, AK

King Salmon, AK 
Dimension Stone

A-K CONSTRUCTION CO.

Ornamental Stone

ANDERSON CONSTRUCTION

BRECHAN ENTERPRISES, INC.

Riprap, Armor, Gabion, or Drain Rock

A-K CONSTRUCTION CO.

ANDERSON CONSTRUCTION

BRECHAN ENTERPRISES, INC.

F/W MATERIALS

LLOYD MOTHERSHEAD

MOORCROFT CONSTRUCTION CO.

\section{Marble}

No respondents.

\section{Limestone or Travertine}

No respondents.

Lime

No respondents.

\section{Gypsum}

No respondents.

Rarite

No respondents.

\section{Horticultural Peat}

ANDERSON CONSTRUCTION

Potting Clays

No respondents.

\section{Top Soil}

A-K CONSTRUCTION CO.

ANDERSON CONSTRUCTION

F/W MATERIALS
Kodiak, AK

Kodiak, AK

Kodiak, AK

Kodiak, AK

Kodiak, AK

Kodiak, AK

Kodiak, AK

Dutch Harbor, AK

King Salmon, AK

Light-weight Aggregate

No respondents.

Kodiak, AK

Kodiak, AK

Kodiak, AK

Kodiak. AK 


\section{Miscellaneous Fill Materials}

ANDERSON CONSTRUCTION

F/W MATERIALS

LLOYD MOTHERSHEAD

MOORCROFT CONSTRUCIION CO

Kodiak, AK

Kodiak, AK

Dutch Harbor, AK

King Salmon, AK

\section{Byproducts}

No respondents.

\section{Southcentral Region}

\section{Pit-run Gravel}

ALYESKA MGT. SERVICES

ASSOCIATED CONSTRUCTION

CASWELL CREEK SAND \& GRAVEL

CENTRAL PAVING PRODUCTS

D.C. RAPPE EXCAVATING

DIBBLE CREEK ROCK

ED'S GRAVEL PIT

FLETCHER EXCAVATING

GLANVILLE LUMBER CO.

HARRIS SAND \& GRAVEL, INC.

JACKSON CONSTRUCTION COMPANY

JIM CLINE'S ENTERPRISES

KLONDIKE-ALASKA, INC.

LUKE'S MINING CO.

MERKES BUILDERS UNLIMITED

MOFFITT \& DAUGHTERS

MORGAN ENTERPRISES

NEAL \& COMPANY

NORTHWIND ENTERPRISES

PATE CONSTRUCTION

Q11

QUALITY SAND \& GRAVEL

RESOURCE EXPLORATION \& DEVELOPMENT

SANDVIK ENTERPRISES

SHULTZ EXCAVATING

SPECIALTY EXCAVATING

THE DIRT COMPANY

WITMAN ENTERPRISES

Girdwood, AK

Anchor Point, AK

Willow, AK

Anchorage, AK

Nikiski, AK

Anchor Point, AK

Kenai, AK

Soldotna, AK

Anchor Point, AK

Valdez, AK

Soldotna, AK

Glennallen, AK

Chugiak, AK

Homer, AK

Soldotna, AK

Palmer, AK

Soldotna, AK

Homer, AK

Gakona, AK

Yakutat, AK

Glennallen, AK

Palmer, AK

Palmer, AK

Palmer, AK

Fairbanks, AK

Soldotna, AK

Big Lake, AK

Eagle River, AK

Screened Aggregate

CIC, INC. 


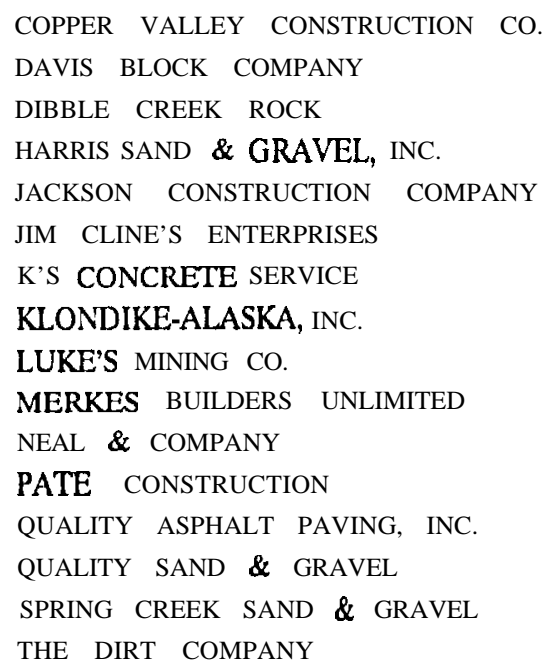

\section{Concrete Gravel and Sand}

ASSOCIATED CONSTRUCTION

CENTRAL PAVING PRODUCTS

COPPER VALLEY CONSTRUCTION CO.

DAVIS BLOCK COMPANY

DIBBLE CREEK ROCK

ED'S GRAVEL PIT

FLETCHER EXCAVATING

HARRIS SAND \& GRAVEL, INC.

JACKSON CONSTRUCTION COMPANY

JIM CLINE'S ENTERPRISES

K'S CONCRETE SERVICE

KLONDIKB-ALASKA, INC.

KNIK GRAVEL

LUKE'S MINING CO.

NEAL \& COMPANY

NELSON SAND \& GRAVEL

PATE CONSTRUCTION

\section{Q11}

QUALITY SAND \& GRAVEL

RESOURCE EXPLORATION \& DEVELOPMENT

ROCK PRODUCTS, INC.

THE DIRT COMPANY

WASILLA CONCRETE!

WILDER CONSTRUCTION CO. INC.

Glennallen, AK

Soldotna, AK

Anchor Point, AK

Valdez, AK

Soldotna, AK

Glennallen, AK

Homer, AK

Chugiak, AK

Homer, AK

Soldotna, AK

Homer, AK

Yakutat, AK

Anchorage, AK

Palmer, AK

Chugiak, AK

Big Lake, AK

Anchor Point, AK

Anchorage, AK

Glennallen, AK

Soldotna, AK

Anchor Point, AK

Kenai, AK

Soidotna, AK

Valdez, AK

Soldotna, AK

Glennallen, AK

Homer, AK

Chugiak, AK

Wasilla, AK

Homer, AK

Homer, AK

Willow, AK

Yakutat, AK

Glennallen, AK

Palmer, AK

Palmer, AK

Wasilla, AK

Big Lake, AK

Wasilia, AK

Anchorage, AK 


\section{Ready-mix Concrete \\ COPPER VALLEY CONSTRUCTION CO. DAVIS BLOCK COMPANY \\ HARRIS SAND \& GRAVEL, INC. \\ K'S CONCRETE SERVICE \\ KLONDIKE-ALASKA, INC. \\ PATE CONSTRUCTION \\ QUALITY SAND \& GRAVEL \\ U.S. CEMENT COMPANY \\ WASILLA CONCRETE}

Concrete Blocks and Similar Products

DAVIS BLOCK COMPANY

K'S CONCRETE SERVICE

\section{Pavement Products}

CENTRAL PAVING PRODUCTS

QUALITY ASPHALT PAVING, INC.

QUALITY SAND \& GRAVEL

RESOURCE EXPLORATION \& DEVELOPMENT

TIDEMARK CORPORATION

WILDER CONSTRUCTION CO. INC.

\section{Asphalt Gravel and Sand}

ASSOCIATED CONSTRUCTION

CENTRAL PAVING PRODUCTS

CIC, INC

HERMON BROTHERS CONSTRUCTION CO., INC.

LUKE'S MINING CO.

MERKES BUILDERS UNLIMITED

NEAL \& COMPANY

NELSON SAND \& GRAVEL

PATE CONSTRUCTION

QUALITY ASPHALT PAVING, INC.

SPECIALTY EXCAVATING

TIDEMARK CORPORATION

WILDER CONSTRUCTION CO. INC.

\section{Masonry-mortar Sand}

CASWELL CREEK SAND \& GRAVEL

DAVIS BLOCK COMPANY

DIBBLE CREEK ROCK

HARRIS SAND \& GRAVEL, INC.
Glennallen, AK

Soldotna, AK

Valdez, AK

Homer, AK

Chugiak, AK

Yakutat, AK

Palmer, AK

Anchorage, AK

Wasilla. AK

Soldotna, AK

Homer, AK

Anchorage, AK Anchorage, AK Palmer, AK Palmer, AK Homer, AK Anchorage, AK

Anchor Point, AK Anchorage, AK Soldotna, AK Palmer, AK Homer, AK Soldotna, AK Homer, AK Willow, AK Yakutat, AK Anchorage, AK Soldotna, AK Homer, AK Anchorage, AK

Willow, AK Soldotna, AK Anchor Point, AK Valdez, AK 
PRODUCT LISTING-SOUTICENTRA L REGION

JACKSON CONSTRUCTION COMPANY

K'S CONCRETE! SERVICE

LUKE'S MINING CO.

NEAL \& COMPANY

QUALITY SAND \& GRAVEL

\section{Masonry-grout Aggregate}

DAVIS BLOCK COMPANY

DIBBLE CREEK ROCK

HARRIS SAND \& GRAVEL, INC.

JACKSON CONSTRUCTION COMPANY

K'S CONCRETE SERVICE

LUKE'S MINING CO.

NEAL \& COMPANY

\section{Built-up Roof Aggregate}

LUKE'S MINING CO.

NEAL \& COMPANY

\section{Filter Sand}

ASSOCIATED CONSTRUCTION

CASWELL CREEK SAND \& GRAVEL

DIBBLE CREEK ROCK

K'S CONCRETE SERVICE

KNIK GRAVEL

LUKE'S MINING CO.

NEAL \& COMPANY

QUALITY SAND \& GRAVEL

SPECIALTY EXCAVATING

SPRING CREEK SAND \& GRAVEL

THE DIRT COMPANY

\section{Blasting Sand}

NELSON SAND \& GRAVEL

\section{Glass Sand}

No respondents.

\section{Furnace Slag}

No respondents.

\section{Crushed Rock}

ASSOCIATED CONSTRUCTION

CENTRAL PAVING PRODUCTS
Soldotna, AK

Homer, AK

Homer, AK

Homer, AK

Palmer, AK

Soldotna, AK

Anchor Point, AK

Valdez, AK

Soldotna, AK

Homer, AK

Homer, AK

Homer, AK

Homer, AK

Homer, AK

Anchor Point, AK

Willow, AK

Anchor Point, AK

Homer, AK

Wasilla, AK

Homer, AK

Homer, AK

Palmer, AK

Soldotna, AK

Chugiak, AK

Big Lake, AK

Willow, AK 


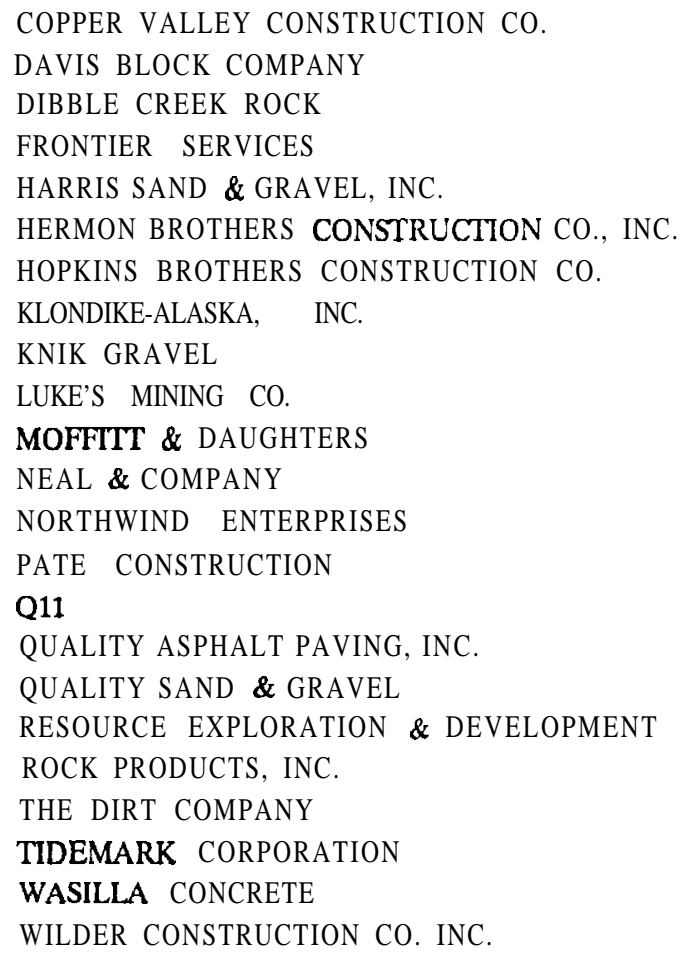

\section{Dimension Stone}

ALASKA MARBLE \& GRANITE ASSOCIATED CONSTRUCTION FRONTIER SERVICES KLONDIKE-ALASKA, INC. MERKES BUILDERS UNLIMITED QUALITY SAND \& GRAVEL

\section{Ornamental Stone}

ALASKA MARBLE \& GRANITE FRONTIER SERVICES INNOKO RIVER ENTERPRISES KLONDIKE-ALASKA, INC. PATE CONSTRUCTION

\section{Riprap, Armor, Gabion, or Drain Rock} ASSOCIATED CONSTRUCTION CENTRAL PAVING PRODUCTS COPPER VALLEY CONSTRUCTION CO.
Glennallen, AK Soldotna, AK Anchor Point, AK Hope, AK Valdez, AK Palmer, AK Seldovia, A K Chugiak, AK Wasilla, AK Homer, AK Palmer, AK Homer, AK Gakona, AK Yakutat, AK Glennallen, AK Anchorage, AK Palmer, AK Palmer, AK Wasilla, AK Big Lake, AK Homer, AK Wasilla, AK Anchorage, AK

Anchorage, AK Anchor Point, AK Hope, AK Chugiak, AK Soldotna, AK Palmer, AK

Anchorage, AK Hope, AK Wasilla, AK Chugiak, AK Yakutat, AK

Anchor Point, AK Anchorage, AK Glennallen. AK 
D.C. RAPPE EXCAVATING

DAVIS BLOCK COMPANY

DIBBLE CREEK ROCK

FRONTIER SERVICES

HOPKINS BROTHERS CONSTRUCTION CO.

JACKSON CONSTRUCTION COMPANY

JIM CLINE'S ENTERPRISES

KI.ONDIKE-ALASKA, INC.

KNIK GRAVEL

LUKE'S MINING CO.

MERKES BUILDERS UNLIMITED

MOFFITT \& DAUGHTERS

MORGAN ENTERPRISES

NEAL \& COMPANY

NORTHWIND ENTERPRISES

Q11

QUALITY SAND \& GRAVEL

RESOURCE EXPLORATION \& DEVELOP.

ROCK PRODUCTS, INC.

SANDVIK ENTERPRISES

SPECIALTY EXCAVATING

SPRING CREEK SAND \& GRAVEL

THE DIRT COMPANY

TIDEMARK CORPORATION

\section{Marble}

ALASKA MARBLE \& GRANITE

Limestone or Travertine

ALASKA MARBLE \& GRANITE

FRONTIER SERVICES

Lime

No respondents.

\section{Gypsum}

No respondents.

\section{Barite}

No respondents.
Nikiski, AK

Soldotna, AK

Anchor Point, AK

Hope, AK

Seldovia, AK

Soldotna, AK

Glennallen, AK

Chugiak, AK

Wasilla, AK

Homer, AK

Soldotna, AK

Palmer, AK

Soldotna, AK

Homer, AK

Gakona, AK

Glennallen, AK

Palmer, AK

Palmer, AK

Wasilla, AK

Palmer, AK

Soldotna, AK

Chugiak, AK

Big Lake, AK

Homer, AK

Anchorage, AK

Anchorage, AK

Hope, AK 


\section{Horticultural Peat}

ED'S GRAVEL PIT

LANDSCAPE SUPPLY CORPORATION

Q11

Kenai, AK

Wasilla, AK

Glennallen, AK

\section{Potting Clays}

FRONTIER SERVICES

Hope, AK

\section{Top Soil}

CREMER SERVICES

D.C. RAPPE EXCAVATING

DAVIS BLOCK COMPANY

DIBBLE CREEK ROCK

ED'S GRAVEL PIT

FLETCHER EXCAVATING

HERMON BROTHERS CONSTRUCTION CO., INC.

HOPKINS BROTHERS CONSTRUCTION CO.

JACKSON CONSTRUCTION COMPANY

JIM CLINE'S ENTERPRISES

LANDSCAPE SUPPLY CORPORATION

LUKE'S MINING CO.

MERKES BUILDERS UNLIMITED

MORGAN ENTERPRISE-S

NEAL \& COMPANY

Q11

QUALITY SAND \& GRAVEL

RESOURCE EXPLORATION \& DEVELOPMENT

SHULTZ EXCAVATING

SPECIALTY EXCAVATING

THE DIRT COMPANY

TIDEMARK CORPORATION

Light-weight Aggregate

FRONTIER SERVICES

\section{Miscellaneous Fill Materials}

ALYESKA MANAGEMENT SERVICES

ASSOCIATED CONSTRUCTION

CASWELL CREEK SAND \& GRAVEL

CENTRAL PAVING PRODUCTS

CREMER SERVICES

D.C. RAPPE EXCAVATING

DIBBLE CREEK ROCK

Hope, AK

Wasilla, AK

Nikiski, AK

Soldotna, AK

Anchor Point, AK

Kenai, AK

Soldotna, AK

Palmer, AK

Seldovia, AK

Soldotna, AK

Glennallen, AK

Wasilla, AK

Homer, AK

Soldotna, AK

Soldotna, AK

Homer, AK

Glennallen, AK

Palmer, AK

Palmer, AK

Fairbanks, AK

Soldotna, AK

Big Lake, AK

Homer, AK

Girdwood, AK

Anchor Point, AK

Willow, AK

Anchorage, AK

Wasilla, AK

Nikiski, AK

Anchor Point, AK 
HARRIS SAND \& GRAVEL, INC.

HERMON BROTHERS CONSTRUCTION CO., INC.

JACKSON CONSTRUCTION COMPANY

JIM CLINE'S ENTERPRISES

KLONDIKE-ALASKA, INC.

KNIK GRAVEL

LUKE'S MINING CO.

MERKES BUILDERS UNLIMITED

MOFFITT \& DAUGHTERS

NEAL \& COMPANY

Q11

QUALITY ASPHALT PAVING, INC.

QUALITY SAND \& GRAVEL

SHULTZ EXCAVATING

SPECIALTY EXCAVATING

TIDEMARK CORPORATION

\section{Byproducts}

FRONTIER SERVICES

INNOKO RIVER ENTERPRISES

\section{Southeastern Region}

\section{Pit-run Gravel}

BRUCE MORLEY

JUNEAU READY MIX, INC.

NORTHERN TIMBER CORPORATION

RITCHIE TRANSPORTATION CO., INC.

\section{Screened Aggregate}

JUNEAU READY MIX. INC.

\section{Concrete Gravel and Sand}

JUNEAU READY MIX, INC. NORTHERN TIMBER CORPORATION RITCHIE TRANSPORTATION CO., INC.

SITKA READY-MIX, INC.

WALDO ENTERPRISES

Ready-mix Concrete DOUBLE "S" CONCRETE

JUNEAU READY MIX, INC.

RITCHIE TRANSPORTATION CO., INC.
Valdez, A K

Palmer, AK

Soldotna, AK

Glennallen, AK

Chugiak, AK

Wasilla, A K

Homer, AK

Soldotna, AK

Palmer, AK

Homer, AK

Glennallen, AK

Anchorage, AK

Palmer, AK

Fairbanks, AK

Soldotna, AK

Homer, AK

Hope, AK

Wasilla, AK
Juneau, AK

Juneau, AK

Haines, AK

Wrangell, AK

Juneau, AK

Juneau, AK

Haines, AK

Wrangell, AK

Sitka, AK

Haines. AK

Haines, AK

Juneau, AK

Wrangell, AK 
SITKA READY-MIX, INC.

WALDO ENTERPRISES

\section{Concrete Blocks and Similar Products}

JUNEAU READY MIX, INC.

\section{Pavement Products}

JUNEAU ASPHALT

NORTHERN TIMBER CORPORATION

Asphalt Gravel and Sand

JUNEAU READY MIX, INC.

NORTHERN TIMBER CORPORATION

Masonry-mortar Sand

JUNEAU READY MIX, INC.

Masonry-grout Aggregate

JUNEAU READY MIX. INC.

\section{Built-up Roof Aggregate}

JUNEAU READY MIX, INC.

\section{Filter Sand}

JUNEAU ASPHALT

JUNEAU READY MIX, INC.

\section{Blasting Sand}

JUNEAU READY MIX, INC.

SITKA READY-MIX, INC.

\section{Glass Sand}

No respondents.

Furnace Slag

JUNEAU READY MIX. INC.

\section{Crushed Rock}

HAINES ENTERPRISES

J.S. CONSTRUCTION, INC.

JUNEAU ASPHALT

JUNEAU READY MIX, INC.

KADIN CORPORATION
Sitka, AK

Haines, AK

Juneau, AK

Juneau, AK

Haines, AK

Juneau, AK

Haines, AK

Juneau, AK

Juneau, AK

Juneau, AK

Juneau, AK

Juneau, AK

Juneau, AK

Sitka, AK
Wrangell, AK

Craig, AK

Juneau, AK

Juneau, AK

Wrangell, AK 
NORTHERN TIMBER CORPORATION

ROCK \& ROAD CONSTRUCTION

\section{Dimension Stone}

HAINES ENTERPRISES

HARD ROCK, INC.

HIGH DRIVE DRILLING \& BLASTING

IIOWARD C. BRAND \&SON

J.S. CONSTRUCTION, INC.

JUNEAU READY MIX, INC.

KIANA CORPORATION

\section{Ornamental Stone}

HARD ROCK, INC.

KIANA CORPORATION

Riprap, Armor, Gabion, or Drain Rock

HAINES ENTERPRISES

HARD ROCK, INC.

HOWARD C. BRAND \& SON

J.S. CONSTRUCTION, INC.

JUNEAU ASPHALT

JUNEAU READY MIX, INC.

KADIN CORPORATION

KIANA CORPORATION

NORTHERN TIMBER CORPORATION

RITCHIE TRANSPORTATION CO., INC.

ROCK \& ROAD CONSTRUCTION
Haines, AK

Petersburg, AK

Wrangell, AK

Haines, A K

Sitka, AK

Ketchikan, AK

Craig, AK

Juneau, AK

Haines, AK

Haines, AK

Haines, AK

Wrangell, AK

Haines, AK

Ketchikan, AK

Craig, AK

Juneau, AK

Juneau, AK

Wrangell, AK

Haines, AK

Haines, AK

Wrangell, AK

Petersburg, AK

\section{Marble}

No respondents.

\section{Limestone or Travertine}

No respondents.

\section{Lime}

No respondents.

\section{Gypsum}

No respondents.

Rarite

`oo respondents. 


\title{
INFORMATION CIRCULA $R 32$
}

\section{Horticultural Peat}

\author{
No respondents.
}

\section{Potting Clays}

No respondents.

\section{Top Soil}

BRUCE MORLEY

HAINES ENTERPRISES

HARD ROCK, INC.

HOWARD C. BRAND \&SON

JUNEAU READY MIX, INC.

KADIN CORPORATION

KIANA CORPORATION

NORTHERN TIMBER CORPORATION

Light-weight Aggregate

No respondents.

\section{Miscellaneous Fill Materials}

JUNEAU READY MIX, INC.

Juneau, AK Wrangell, AK Haines, AK Ketchikan, AK Juneau, AK Wrangell, AK

Haines, A K Haines, AK

Juneau, AK

\section{Byproducts}

No respondents.

\section{Out-of-state Region}

\section{Pit-run Gravel}

MARINE CONSTRUCTION \& DREDGING, INC.

Mt Vernon, WA

Screened Aggregate

MARINE CONSTRUCTION \& DREDGING, INC.

Mt Vernon, WA

Concrete Gravel and Sand MARINE CONSTRUCTION \& DREDGING, INC.

Mt Vernon, WA

\section{Ready-mix Concrete}

No respondents.

Concrete Blocks and Similar Products

No respondents. 


\section{Pavement Products}

No respondents.

Asphalt Gravel and Sand

No respondents.

\section{Masonry-mortar Sand \\ No respondents.}

\section{Masonry-grout Aggregate}

No respondents.

\section{Built-up Roof Aggregate}

No respondents.

Filter Sand

MARINE CONSTRUCTION\& DREDGING, INC.

Mt Vernon, WA

\section{Blasting Sand}

No respondents.

\section{Glass Sand}

No respondents.

\section{Furnace Slag}

No respondents.

\section{Crushed Rock}

No respondents.

\section{Dimension Stone}

No respondents.

\section{Ornamental Stone}

No respondents.

Riprap, Armor, Gabion, or Drain Rock

No respondents.

\section{Marble}

No respondents. 


\section{Limestone or Travertine}

No respondents.

\section{Lime}

No respondents.

\section{Gypsum}

No respondents.

\section{Barite}

No respondents.

\section{Horticultural Peat}

No respondents.

\section{Potting Clays}

No respondents.

Top Soil

No respondents.

Light-weight Aggregate

No respondents.

\section{Miscellaneous Fill Materials}

MARINE CONSTR \& DREDGING, INC.

Mt Vernon, WA

\section{Byproducts}

No respondents. 
SECTION 2 VENDOR LISTING

\section{Northern Region}

No respondents.

\section{Western Region}

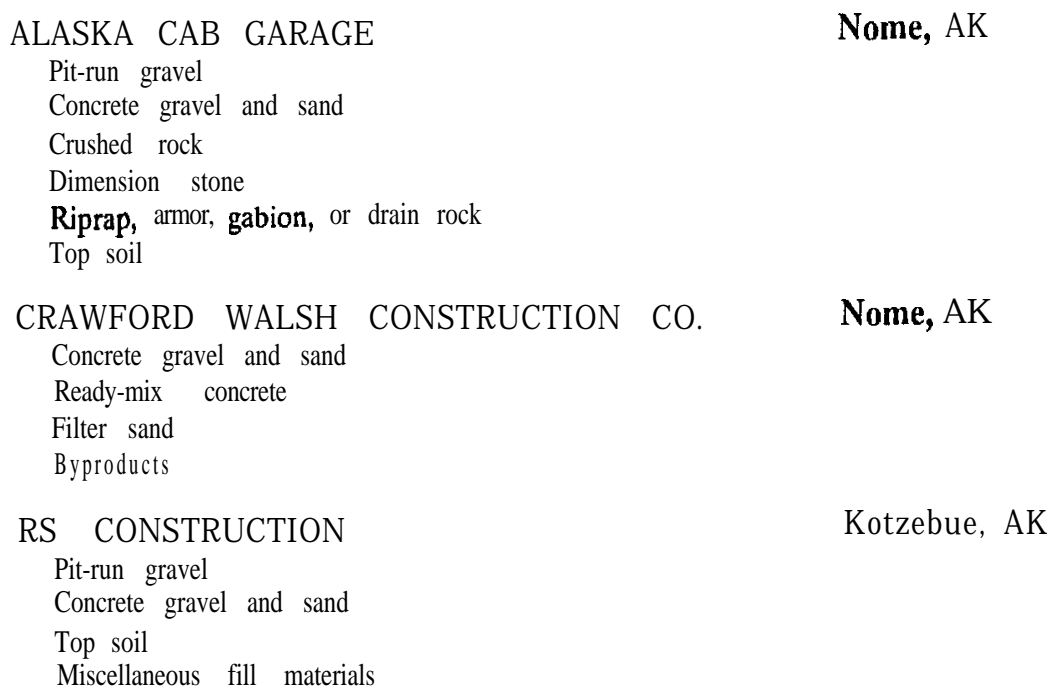

Nome, AK

Kotzebue, AK

Eastern Interior Region

A.C.E. GENERAL CONTRACTORS, INC.

Fairbanks, AK Screened aggregate

Filter sand

Crushed rock

Ornamental stone

Riprap, armor, gabion, or drain rock

\section{ALASKA LIME COMPANY}

Cantwell, AK

Riprap, armor, gabion, or drain rock Limestone or travertine

ARCTIC REDI-MIX, INC.

Screened aggregate

Concrete gravel and sand

Ready-mix concrete

Crushed rock 
DICKSON ENTERPRISES, INC.

Crushed rock

Dimension stone

Riprap, armor, gabion, or drain rock

Top soil

Miscellaneous fill materials

EARTH MOVERS OF FAIRBANKS, INC.

Screened aggregate

Pavement products

Asphalt gravel and sand

Crushed rock

Dimension stone

\section{EARTH PRODUCTS}

Pit-run gravel

Top soil

Miscellaneous fill materials

EVANS INDUSTRIES, INC.

Screened aggregate

Concrete gravel and sand

Ready-mix concrete

Crushed rock

\section{EXCLUSIVE LANDSCAPING \&}

EXCAVATING, INC.

Top soil

\section{FAIRBANKS PUMPING \& THAWING}

Screened aggregate

Concrete gravel and sand

Pavement products

Crushedrock

Riprap, armor, gabion, or drain rock

Top soil

Miscellaneous fill materials

FAIRBANKS SAND \& GRAVEL, INC.

Screened aggregate

Concrete gravel and sand

Ready-mix concrete

Concrete blocks and similar products
Fairbanks, AK

Fairbanks, AK

North Pole, AK

Healy, AK

Fairbanks, AK

North Pole, AK 


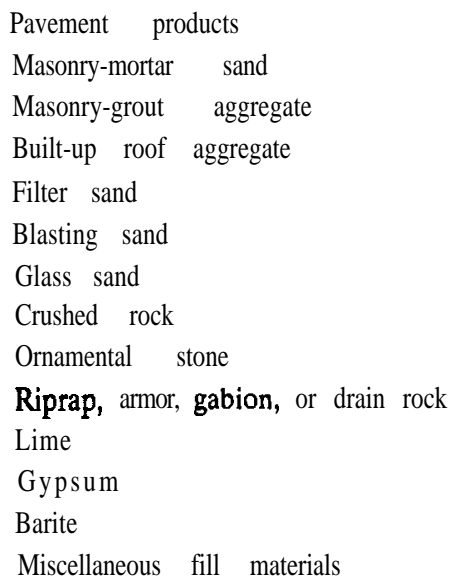

GREAT NORTHWEST, INC.

Screened aggregate

Concrete gravel and sand

Asphalt gravel and sand

Ornamental stone

Horticultural peat

Top soil

Miscellaneous fill materials

II. \& H. CONTRACTORS, INC.

Screened aggregate

Concrete gravel and sand

Ready-mix concrete

Concrete blocks and similar products

Asphalt gravel and sand

Masonry-mortar sand

Masonry-grout aggregate

Filter sand

Crushed rock

Riprap, armor, gabion, or drain rock Horticultural peat

Light-weight aggregate

Miscellaneous fill materials

\section{HOWARD ENTERPRISES}

North Pole, AK

Pit-run gravel

Top soil 


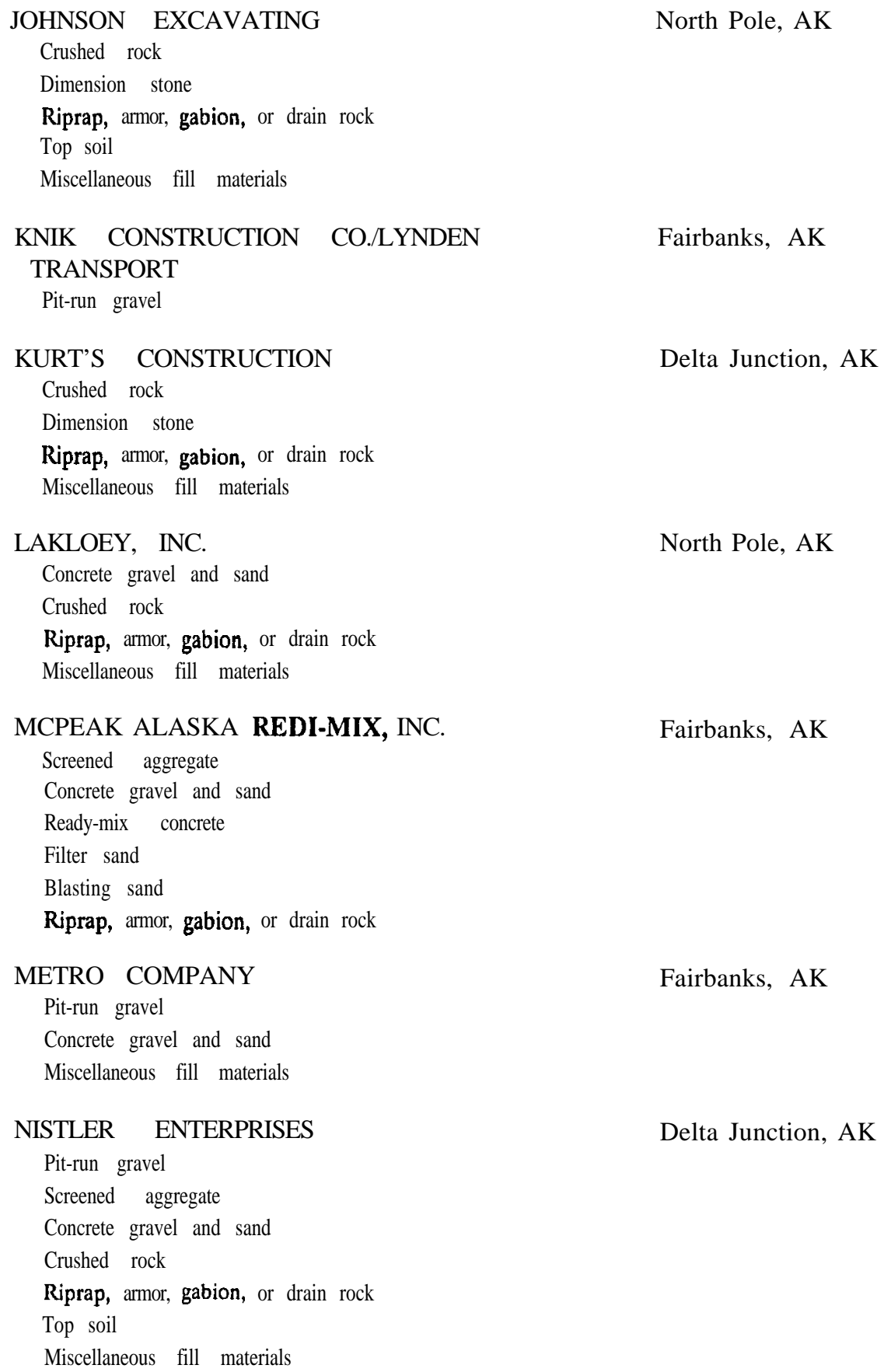

Fairbanks, AK

Delta Junction, AK

North Pole, AK

Fairbanks, AK

Fairbanks, AK

Delta Junction, AK 
VENDOR LISTING-EASTERN INTERIOR REGION

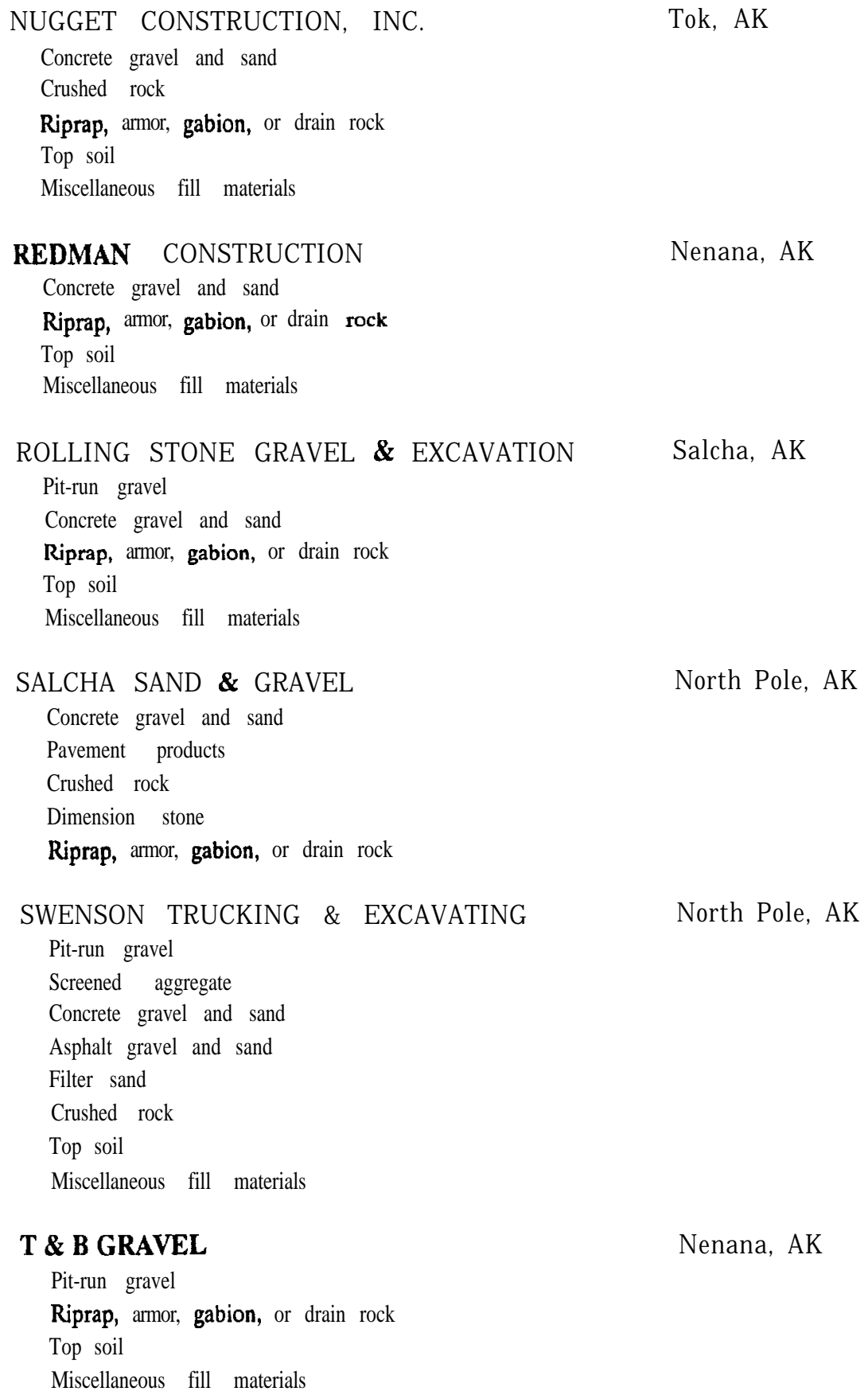

ROLLING STONE GRAVEL \& EXCAVATION Salcha, AK

Nenana, AK

Tok, AK

North Pole, AK

T \& B GRAVEL

Pit-run gravel

Riprap, armor, gabion, or drain rock

Top soil

Miscellaneous fill materials

North Pole, AK 
VENDOR LISTING-ALASKA PENINSULA REGION

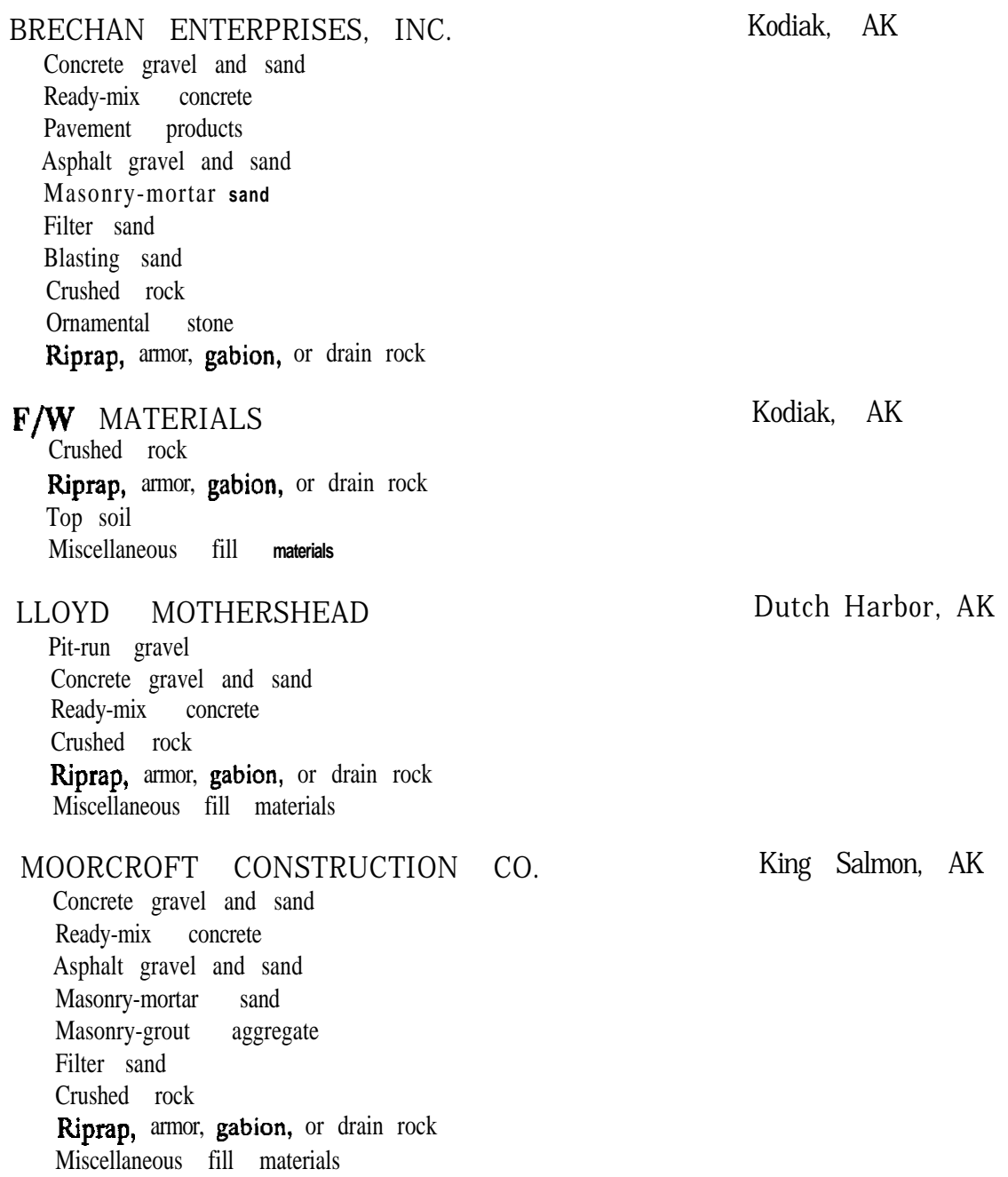

\title{
Southcentral Region
}

\author{
ALASKA MARBLE \& GRANITE \\ Dimension stone \\ Ornamental stone \\ Marble \\ Limestone or travertine \\ ALYESKA MGT. SERVICES \\ Girdwood, AK \\ Anchorage, AK \\ Pit-run gravel \\ Miscellaneous fill materials
}




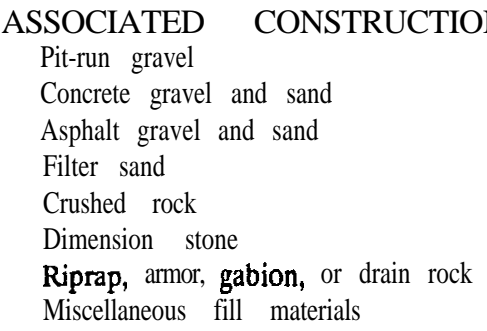

CASWELL CREEK SAND \& GRAVEL

Pit-run gravel

Masonry-mortar

Filter sand

Miscellaneous fill materials

\section{CENTRAL PAVING PRODUCTS \\ Pit-run gravel \\ Concrete gravel and sand \\ Pavement products \\ Asphalt gravel and sand \\ Crushed rock \\ Riprap, armor, gabion, or drain rock \\ Miscellaneous fill materials}

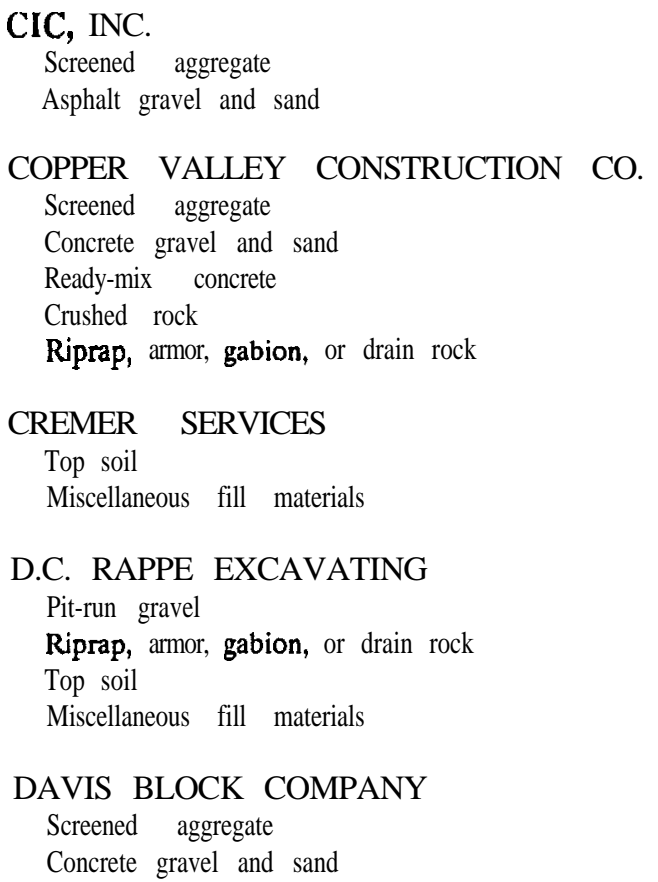

Soldotna, AK

Glennallen, AK
Nikiski, AK

Soldotna, AK 


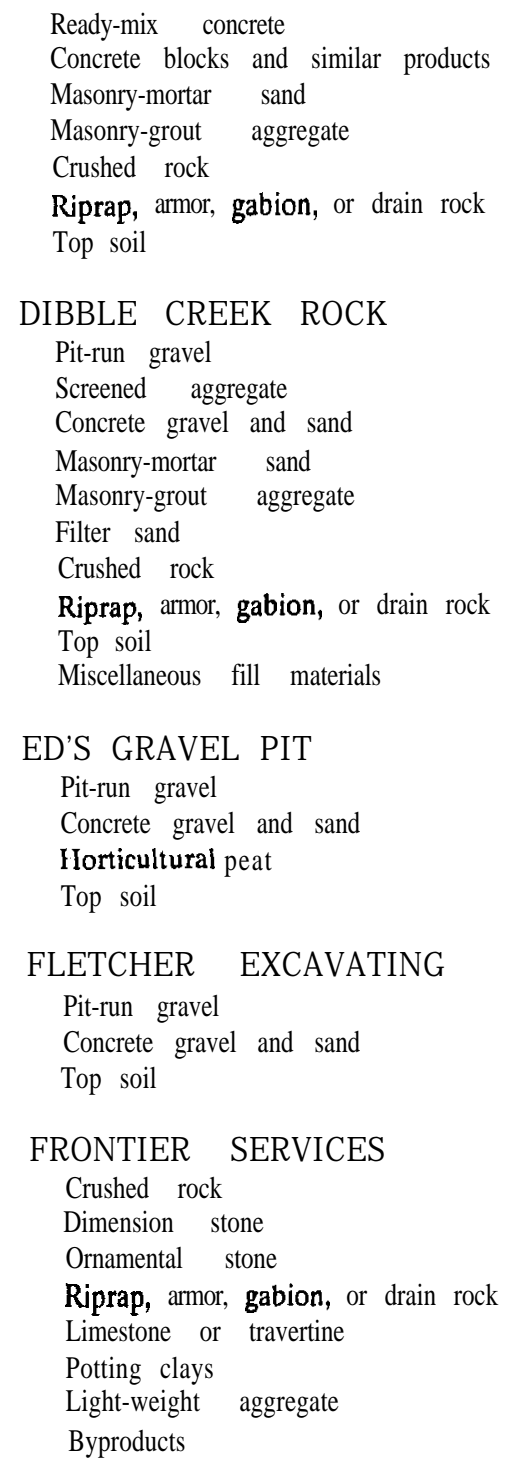

\section{FLETCHER EXCAVATING}

Pit-run gravel Concrete gravel and sand Top soil

\section{FRONTIER SERVICES}

Crushed rock Dimension stone Ornamental stone Riprap, armor, gabion, or drain rock Limestone or travertine Potting clays Light-weight aggregate Byproducts

Anchor Point, AK

GLANVILLE LUMBER CO.

Anchor Point, AK Pit-run gravel

HARRIS SAND \& GRAVEL, INC.

Kenai, AK

Sotdotna, AK

Pit-run gravel

Screened aggregate

Concrete gravel and sand

Ready-mix concrete 


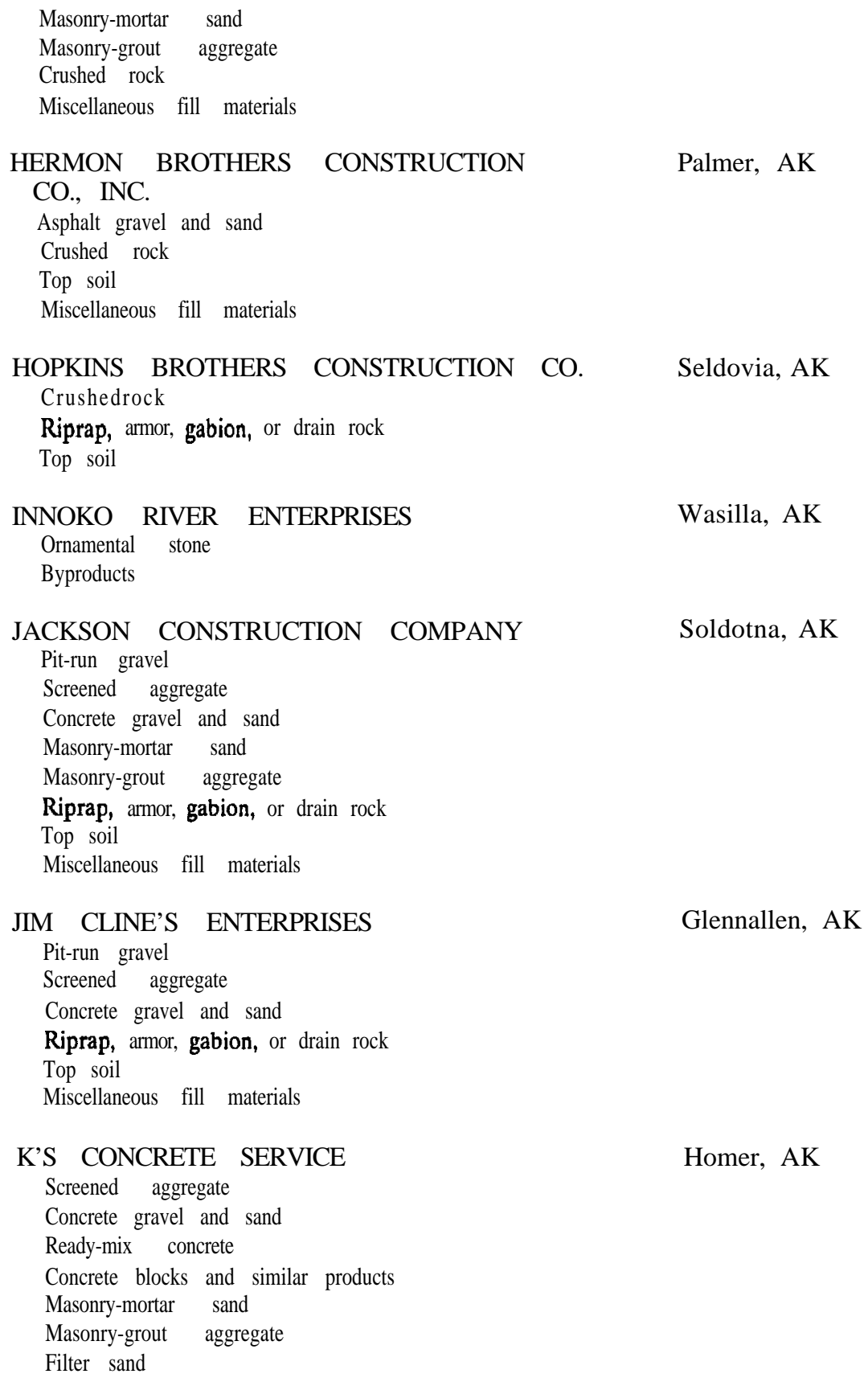


KLONDIKE-ALASKA, INC.

Chugiak, AK

Pit-run gravel

Screened aggregate

Concrete gravel and sand

Ready-mix concrete

Crushed rock

Dimension stone

Ornamental stone

Riprap, armor, gabion, or drain rock

Miscellaneous fill materials

KNIK GRAVEL

Wasilla, AK

Concrete gravel and sand

Filter sand

Crushed rock

Riprap, armor, gabion, or drain rock

Miscellaneous fill materials
LANDSCAPE SUPPLY CORPORATION
Horticultural peat
Top soil

LUKE'S MINING CO.

Pit-run gravel

Screened aggregate

Concrete gravel and sand

Asphalt gravel and sand

Masonry-mortar sand

Masonry-grout aggregate

Built-up roof aggregate

Filter sand

Crushed rock

Riprap, armor, gabion, or drain rock

Top soil

Miscellaneous fill materials

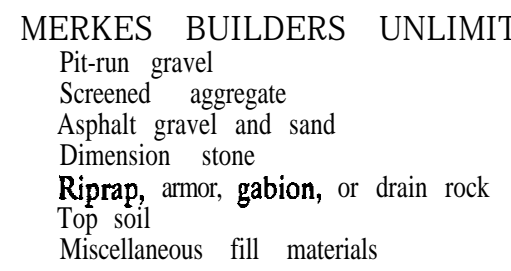

MOFFITT \& DAUGHTERS

Pit-run gravel

Crushed rock

Riprap, armor, gabion, or drain rock

Miscellaneous fill materials 
MORGAN ENTERPRISES

Pit-run gravel

Riprap, armor, gabion, or drain rock Top soil

NEAL \& COMPANY

Pit-run gravel

Screened aggregate

Concrete gravel and sand

Asphalt gravel and sand

Masonry-mortar sand

Masonry-grout aggregate

Built-up roof aggregate

Filter sand

Crushed rock

Riprap, armor, gabion, or drain rock

Top soil

Miscellaneous fill materials

\section{NELSON SAND \& GRAVEL}

Concrete gravel and sand

Asphalt gravel and sand

Blasting sand

\section{NORTHWIND ENTERPRISES \\ Pit-run gravel \\ Crushed rock \\ Riprap, armor, gabion, or drain rock}

PATE CONSTRUCTION

Pit-run gravel

Screened aggregate

Concrete gravel and sand

Ready-mix concrete

Asphalt gravel and sand

Crushed rock

Ornamental stone

\section{Q11}

Pit-run gravel

Concrete gravel and sand

Crushed rock

Riprap, armor, gabion, or drain rock

Horticultural peat

Top soil

Miscellaneous fill materials

QUALITY

Screened

ASPHALT PAVING, INC.

Pavement

aggregate

products
Soldotna, AK

Homer, AK

Willow, AK

Gakona, AK

Yakutat, AK

Glennallen, AK

Anchorage, AK 
LENDOR L I STING-SOU L REG IO N

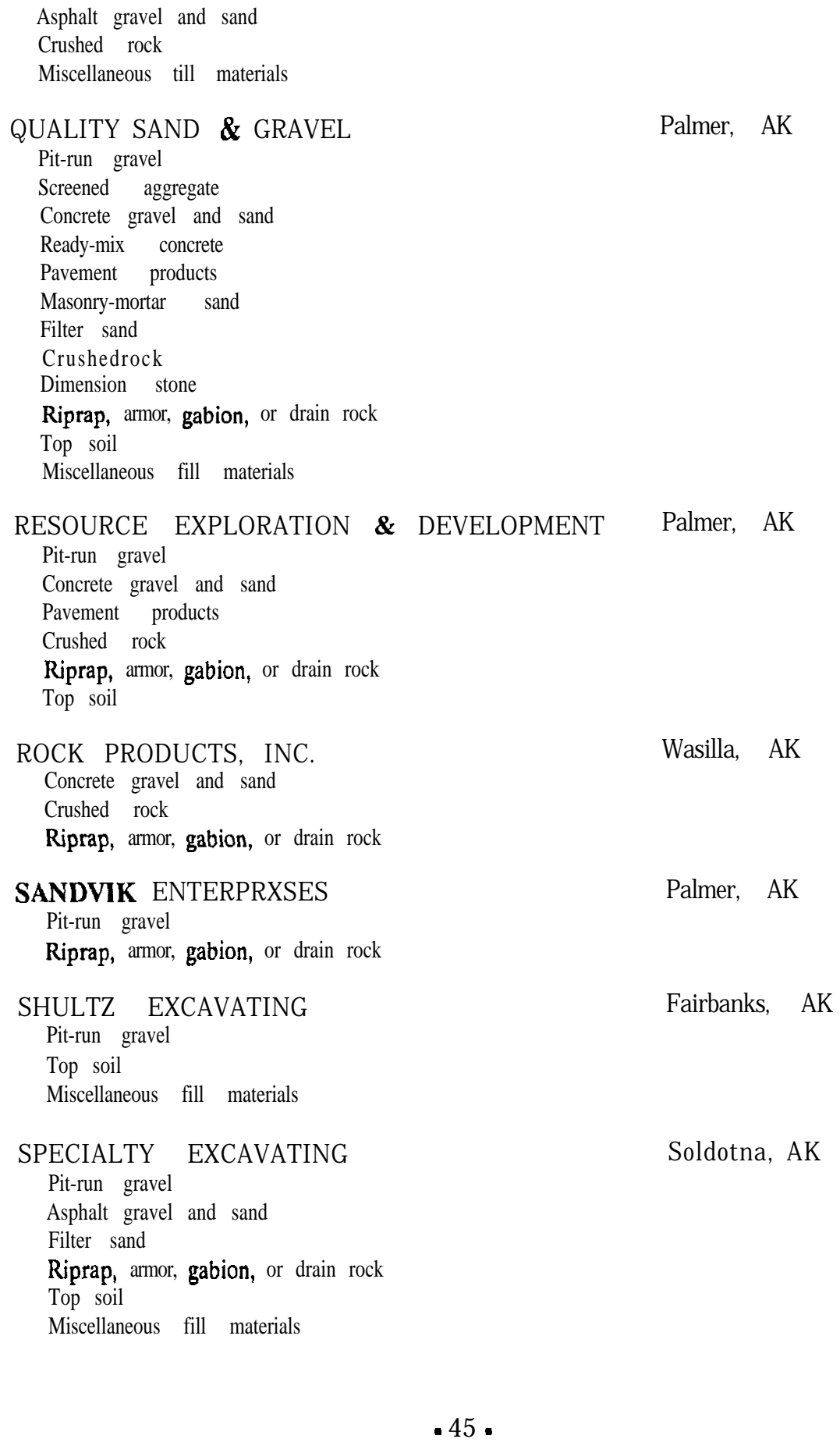




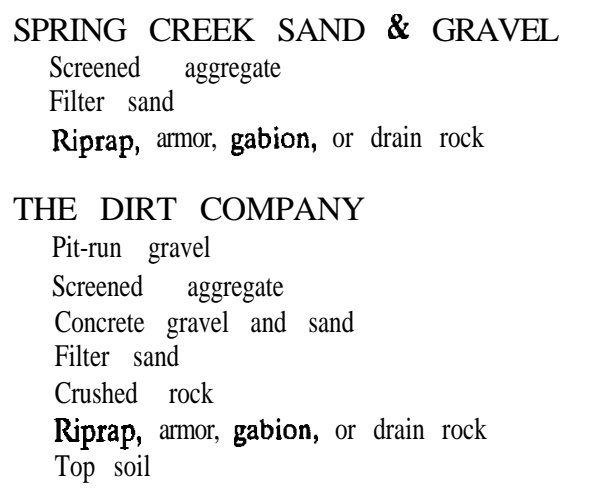

Chugiak, AK

Big Lake, AK

Homer, AK

TIDEMARK CORPORATION

Pavement products

Asphalt gravel and sand

Crushed rock

Riprap, armor, gabion, or drain rock

Top soil

Miscellaneous fill materials

\section{U.S. CEMENT COMPANY}

Ready-mix concrete

WASILLA CONCRETE

Concrete gravel and sand

Ready-mix concrete

Crushed rock

WILDER CONSTRUCTION CO. INC.

Concrete gravel and sand

Pavement products

Asphalt gravel and sand

Crushed rock

WITMAN ENTERPRISES

Pit-run gravel

\section{Southeastern Region}

\author{
BRUCE MORLEY \\ Pit-run gravel \\ Top soil \\ DOUBLE "S" CONCRETE \\ Ready-mix concrete
}

Juneau, AK

Haines, AK
Anchorage, AK

Wasilla, AK

Anchorage, AK

Eagle River, AK 


\section{HAINES ENTERPRISES}

Crushed rock

Dimension stone

Riprap, armor, gabion, or drain rock

Top soil

HARD ROCK, INC.

Haines, AK

Dimension stone

Ornamental stone

Rjprap, armor, gabion, or drain rock

Top soil

\section{HIGH DRIVE DRILLING \& BLASTING}

Dimension stone

HOWARD C. BRAND \& SON

Wrangell, AK

Dimension stone

Riprap, armor, gabion, or drain rock Top soil

\section{J.S. CONSTRUCTION, INC.}

Crushed rock

Dimension stone

Riprap, armor, gabion, or drain rock

JUNEAU ASPHALT

Pavement products

Filter sand

Crushed rock

Riprap, armor, gabion, or drain rock

JUNEAU READY MIX, INC.

Pit-run gravel

Screened aggregate

Concrete gravel and sand

Ready-mix concrete

Concrete blocks and similar products

Asphalt gravel and sand

Masonry-mortar sand

Masonry-grout aggregate

Built-up roof aggregate

Filter sand

Blasting sand

Furnace slag

Crushed rock

Dimension stone

Riprap, armor, gabion, or drain rock

Top soil

Miscellaneous fill materials

Craig, AK

Juneau, AK

Juneau, AK 


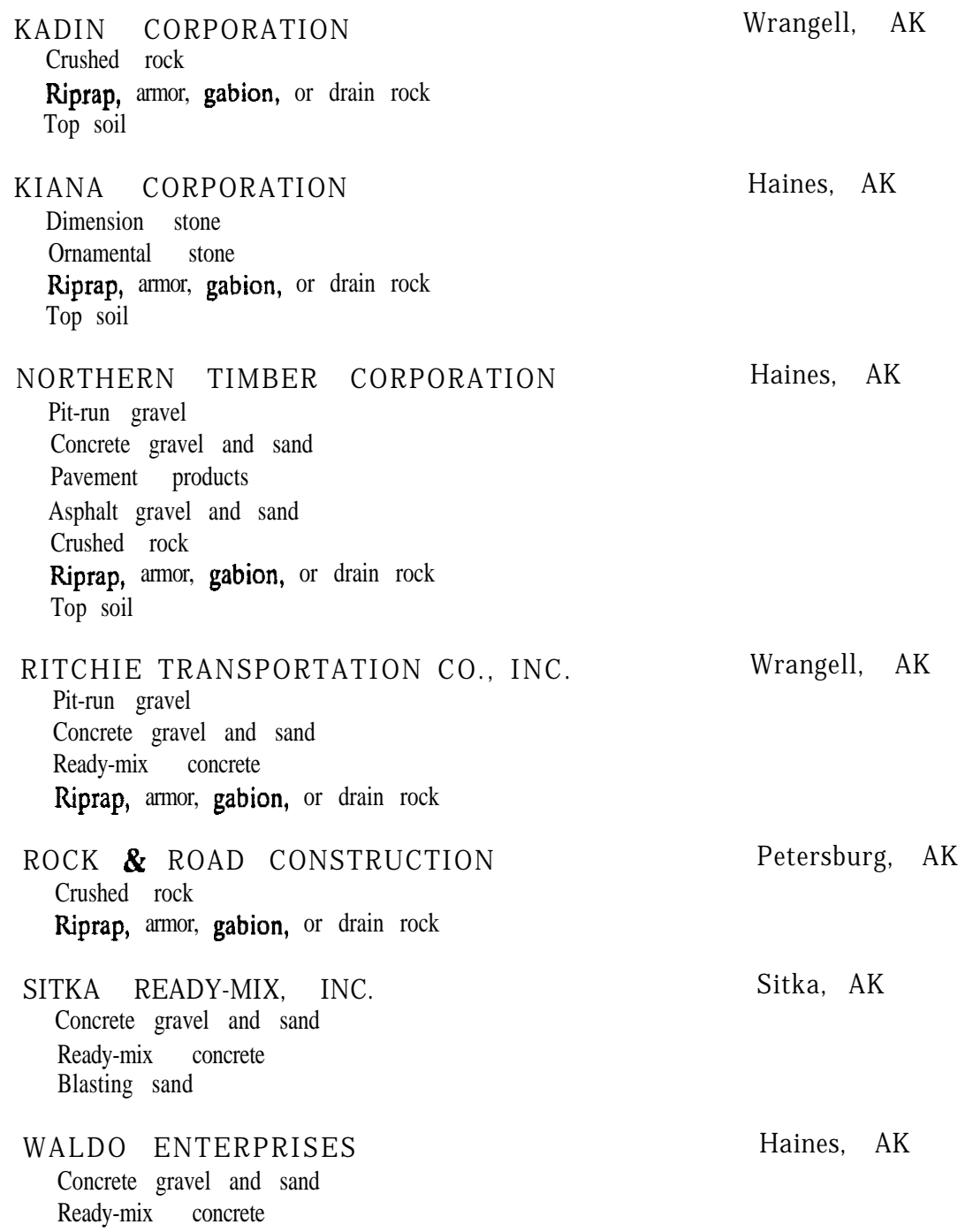

NORTHERN TIMBER CORPORATION Haines, AK

Wrangell, AK

Haines, AK 


\section{SECTION 3 VENDOR ADDRESS LISTING}

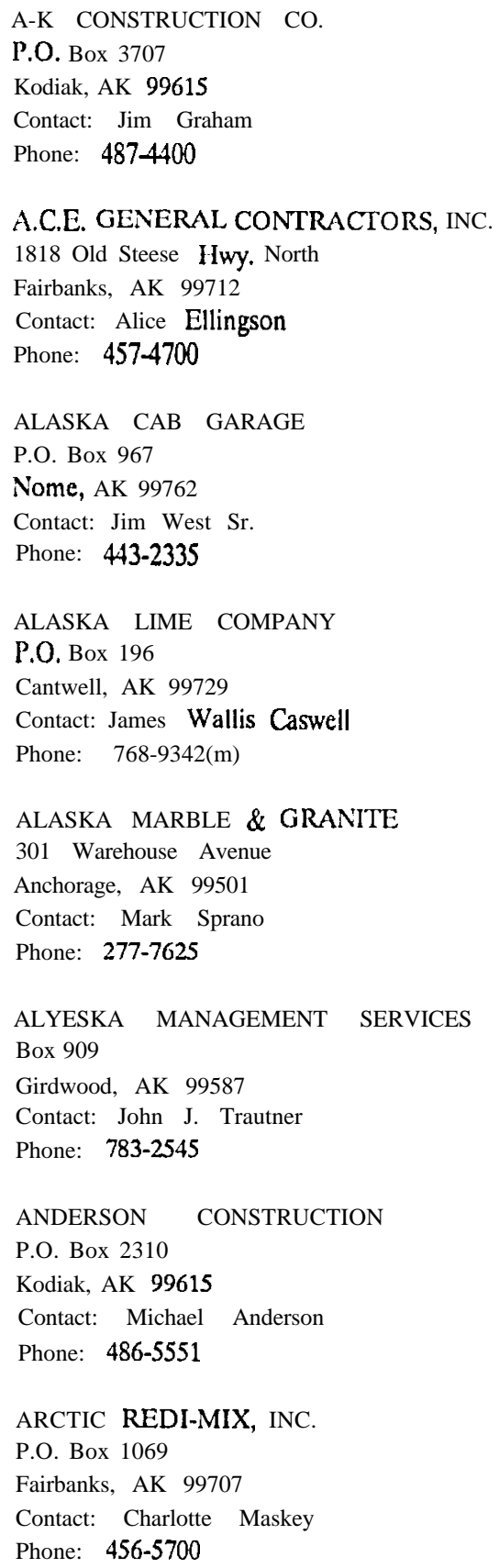


INFORMATION CIRCULAR 32

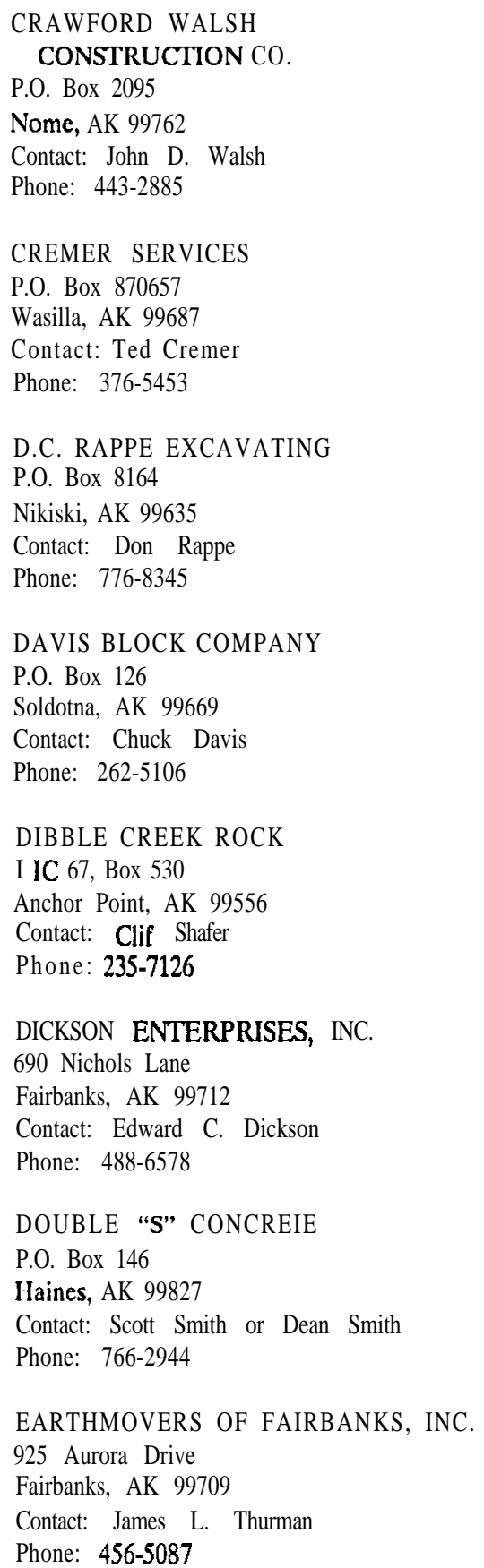

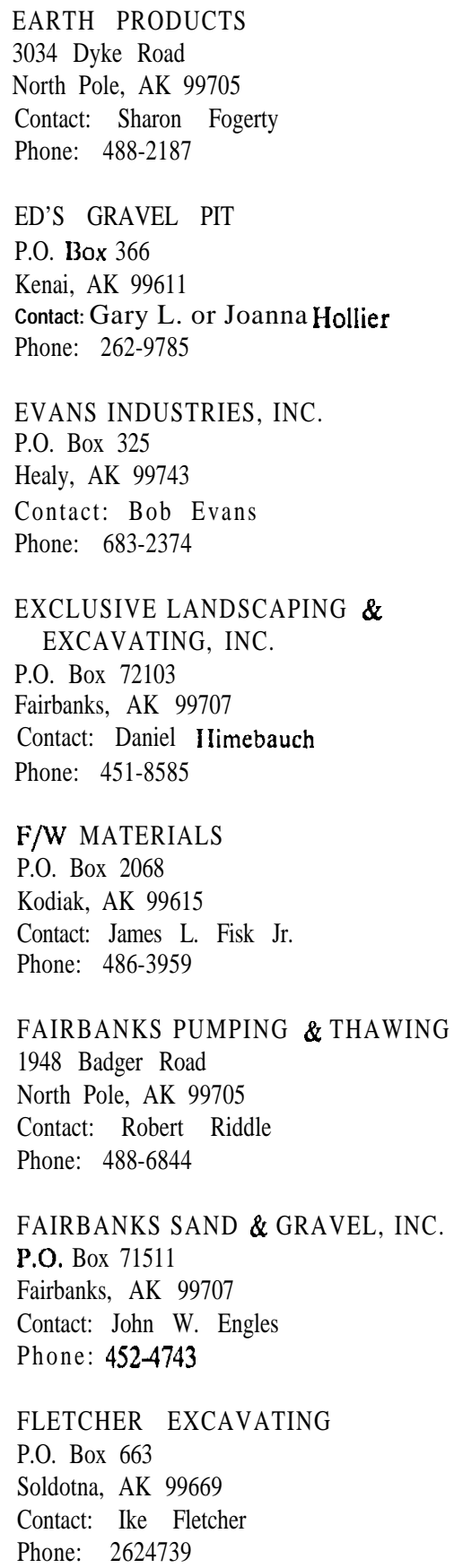




\section{VENDOR ADDRESS LISTING}

FRONTIER SERVICES

P.O. Box 150

I lope, AK 99605

Contact: Roger Moore

Phone: 262-6260

GLANVILLE LUMBER CO.

IIC Box 1195

Anchor Point, AK 99556

Contact: Carl T. Glanville

Phone: 235-8917

GREAT NORTHWEST, INC.

P.O. Box 74646

Fairbanks, AK 99707

Contact: Buzz Otis

Phone: $452-5617$

II. \& H. CONTRACTORS, INC.

Box 60610

Fairbanks, AK 99706

Contact: Larry Horner

Phone: 479-2235

ILAINES ENTERPRISES

P.O. Box 216

Wrangell, AK 99929

Contact: Bill Haines

Phone: $874-3479$

I IARD ROCK, INC

P.O. Box 129

Haines, AK 99827

Contact: Debra J. Schnabel

Phone: 766-2827

HLARRIS SAND \& GRAVEL, INC.

P.O. Box 1347

Valdez, AK 99686

Contact: Bill Harris

Phone: 8354756

I IERMON BROTHERS

CONSTRUCTION CO., INC.

111 West Eimwood

Palmer, AK 99645

Contact: Ben H. Hermon

Phone: $745-3249$
HIGH DRIVE DRILLING \& BLASTING

P.O. Box 2627

Sitka, AK 99835

Contact: Robert \& Eileen Prefontaine

Phone: 747-5724

IIOPKINS BROTHERS

CONSTRUCTION CO.

P.O. Drawer $\mathrm{K}$

Seldovia, AK 99663

Contact: Jim Hopkins

Phone: 234-7843

HOWARD C. BRAND \& SON

8204 South Tongass Hwy.

Ketchikan, AK 99901

Contact: Dennis Brand

Phone: 225-3634

HOWARD ENTERPRISES

P.O. Box 55263

North Pole, AK 99705

Contact: Jack Howard

Phone: 488-6869

INNOKO RIVER ENTERPRISES

P.O. Box 871050

Wasilla, AK 99687

Contact: Ed Gurtler Sr.

Phone: $376-6863$

J.S. CONSTRUCTION, INC.

P.O. Box 79

Craig, AK 99921

Contact: James L. Seley

Phone: 826-3633

JACKSON CONSTRUCTION COMPANY

241 Aspen Street

Soldotna, AK 99669

Contact: Harold A. Jackson

Phone: 262-4485

JIM CLINE'S ENTERPRISES

P.O. Box 2

Glennallen, AK 99588

Contact: James Cline

Phone: $882-3480$ 


\section{INFORMA TION CIRCULAR 3?}

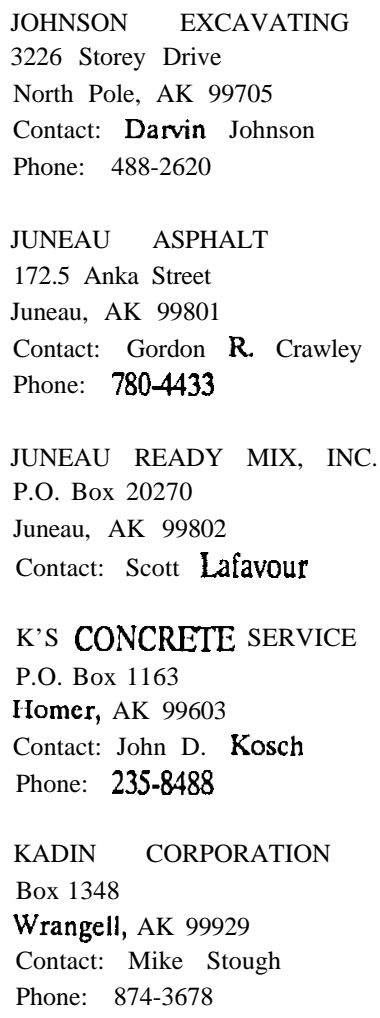

KIANA CORPORATION

P.O. Box 129

I Iaines, AK 99827

Contact: Debra J. Schnabcl

Phone: 766-2827

KLONDIKE-ALASKA, INC.

P.O. Box 671028

Chugiak, AK 99567

Contact: Dick Erickson

Phone: 688-2161

KNIK CONSTRUCTION CO./ LYNDEN TRANSPORT 3001 Peger Road Fairbanks, AK 99701

Contact: Ken Williamson

Phone: 456-5535
KNIK GRAVEL

4900 Palmer-Wasilla Road

Wasilla, AK 99687

Contact: Jim L. McCourt

Phone: 376-9042

KURT'S CONSTRUCTION 1900 Granite View Dr.

Delta Junction, AK 99737

Contact: Kurt Ueeck

Phone: $895-5120$

LAKIOEY, INC.

1216 Range View Road North Pole, AK 99705-5352

Contact: Allen Vezey

Phone: 488-9745

LANDSCAPE SUPPLY CORPORATION 4400 Gray Wolf Drive

Wasilla, AK 99687

Contact: Howard C. Nugent

Phone: 376-3833

\section{LLOYD MOTIIERSHEAD}

P.O. Box 443

Dutch Harbor, AK 99692

Contact: Lloyd Mothershead

Phone: $581-2200$

LUKE'S MINING CO.

2396 Kachemak Drive

Homer, AK 99603

Contact: Tony Neal

Phone: 235-8141

MARINE CONSTRUCTION \& DREDGING, INC.

1579 Dunbar Road

Mt Vernon, WA 98273

Contact: Ken Youngsman

Phone: 206-424-6204

MCPEAK ALASKA REDI-MIX, INC. P.O. Box 58076

Fairbanks, AK 99711

Contact: Velma Leslie

Phone: $488-2388$ 
VENDOR ADDRESS LISTING

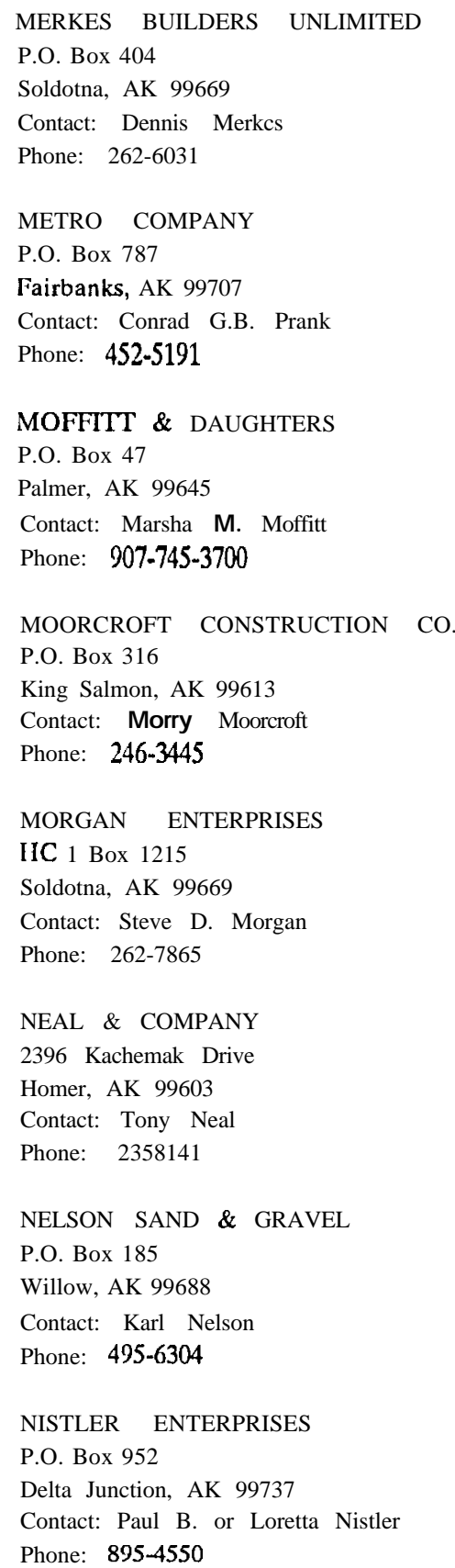

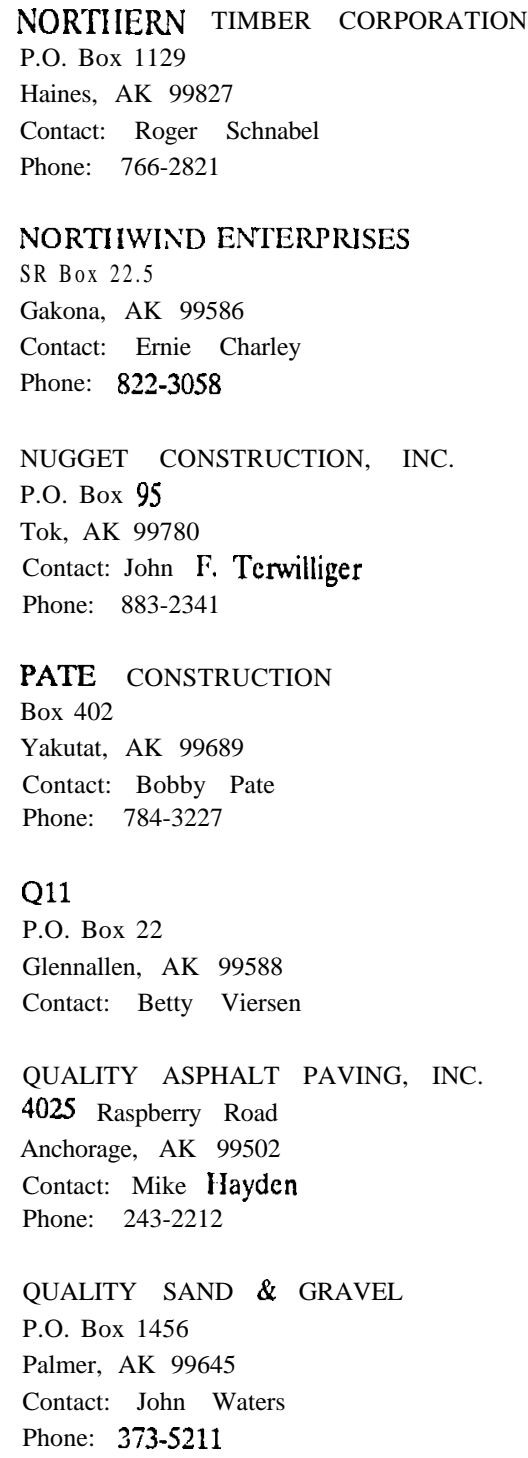

REDMAN CONSTRUCTION P.O. Box 38 Nenana, AK 99760

Contact: Charlie Stevens

Phone: 832-5431 
INFORMATION CIRCULAR 32

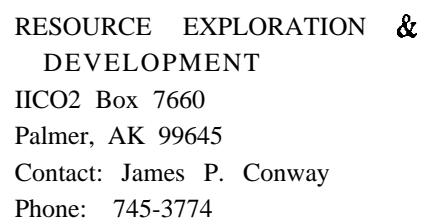

RS CONSTRUCTION

P.O. Box 1010

Kotzebue, AK 99752

Contact: RS Store, Inc.

Phone: 907-442-3653

SALCHA SAND \& GRAVEL

P.O. Box 56159

North Pole, AK 99705

Contact: Gabby Gaborik

Phone: $488-8289$

SANDVIK ENTERPRISES

I IC02 Box 7480-2

Palmer, AK 99645

Contact: Lynn C. Sandvik

Phone: $745-2438$

SHULTZ EXCAVATING

P.O. Box 72233

Fairbanks, AK 99707

Contact: Paul G. Shultz

Phone: 479-2089

SITKA READY-MIX, INC

P.O. Box 880

Sitka, AK 99835

Contact: Don Branham

Phone: 747-8693

\section{SPECIALTY EXCAVATING}

P.O. Box 365

Soldotna, AK 99669

Contact: Jerry Holly

Phone: 262-5175

SPRING CREEK SAND \& GRAVEL

HC78 - Box 1155

Chugiak, AK 99567

Contact: Dixie Waddell

Phone: 688-2610

SWENSON TRUCKING \& EXCAVATING

2005 Richardson Hwy.

North Pole, AK 99705

Contact: Larry Swenson

Phone: 488-6584

T \& B GRAVEL

P.O. Box 294

Nenana, AK 99760

Contact: Donald Beck

Phone: $832-5436$

THE DIRT COMPANY

P.O. Box 520209

Big Lake, AK 99652

Contact: Brad Fisher

Phone: 892-6706

\section{TIDEMARK CORPORATION}

P.O. Box 249

Homer, AK 99603

Contact: Quinten Amdt

Phone: $235-8583$ 
U.S. CEMENT COMPANY

$1400 \mathrm{~W}$. Dowling Road

Anchorage, AK 99518

Contact: LeRoy Dyson

Phone: 563-4228

UNIVERSITY REDI-MIX, INC.

P.O. Box 60750

Fairbanks, AK 99706

Contact: Larry Homer

Phone: 479-2996

WALDO ENTERPRISES

P.O. Box 274

I Iaines, AK 99827

Contact: Bill Waldo

Phone: 766-2631

WASILLA CONCRETE

P.O. Box 870183

Wasilla, AK 99687

Contact: Michael Smith

Phone: 376-5331

WILDER CONSTRUCTION CO. INC

11301 Lang Street

Anchorage, AK 95155-3006

Contact: Paul Ross

Phone: $344-2593$

WITMAN ENTERPRISES

18015 N. Eagle River Lp. Rd.

Eagle River, AK 99577

Contact: Harry or Robert Witman

Phone: 694-2387

WRIGHT'S AURORA CONSTRUCTION

P.O. Box 60515

Fairbanks, AK 99706

Contact: Gareth T. Wright

Phone: $479-4512$

YUTAN CONSTRUCTION CO.

P.O. Box 71775

Fairbanks, AK 99707

Contact: Lewis F. Vondra

Phone: 488-9290 


\section{STAFF'}

Robert B. Forbes, State Geologist

Thomas E. Smith, Deputy State Geologist

\section{Administrative Services}

P.M. Verosta, Administrative Assistant ${ }^{2}$

K.E. Brown, Clerk Typist

D.L. Chavez, Secretary

R.A. Czajka, Clerk Typist

RR Groner, Accounting Tcchnician

M.G. Murphree, Clerk Typist

J.M. Robinson, Administrative Assistant

J.L. Weir, Field Office Assistant

Energy Resources

G.II. Pessel, Geologist'

J.G. Clough, Geologist

S.A. Liss, Geologist

C.G. Mull, Geologist

J.W. Reeder, Geologist

R.R. Reifenstuhl, Geologist

M.S. Robinson, Geologist

\section{Vingineering Geology}

R.A. Combellick, Geologist ${ }^{2}$

G.D. March, Geologist

C.J. Nye, Geologist

R.D. Regcr, Gcologist

M.A. Wiltse, Chemist

Minerals and Materials Development

W.G. Gilbert, Geologist'

N.D. Bowman, Geology Assistant

T.K. Bundtzen, Geologist

L.E. Burns, Geologist

K.H. Clauticc, Geologist

E.E. Harris, Geologist

J.T. Kline, Geologist

D.N. Solic, Geologist
Resource Information

G.M. Laird, Publications Specialist' R.A. Mann, Clerk

J.A. Outten, Publications Technician

A-L. Schell, Cartographer

A.G. Sturmann, Cartographer

F.C. Tannian, Publications Specialist

Water Resources

W.E. Long, Iydrologist ${ }^{2}$

R.D. Allely, Ifydrologist

S.J. Carrick, Hydrologist

M.G. Inghram, Hydrologist

R.W. Ireland, Iydrologist

D.L. LaSage, Hydrologist

M.A. Maurer, Hydrologist

M.A. Moorman, IIydrologist

R.J. Motyka, Geologist

J.A. Munter, IIydrologist

R.S. Noll, Ilydrologist

W,A. Petrik, Ilydrologist

S.R. Ray, Hydrologist

J.J. Vohden, Chemist

1In addition to the permanent staff listed above, DGGS presently employs 14 students in the Department of Natural Resources Student Intern Program.

${ }^{2}$ Section chief. 


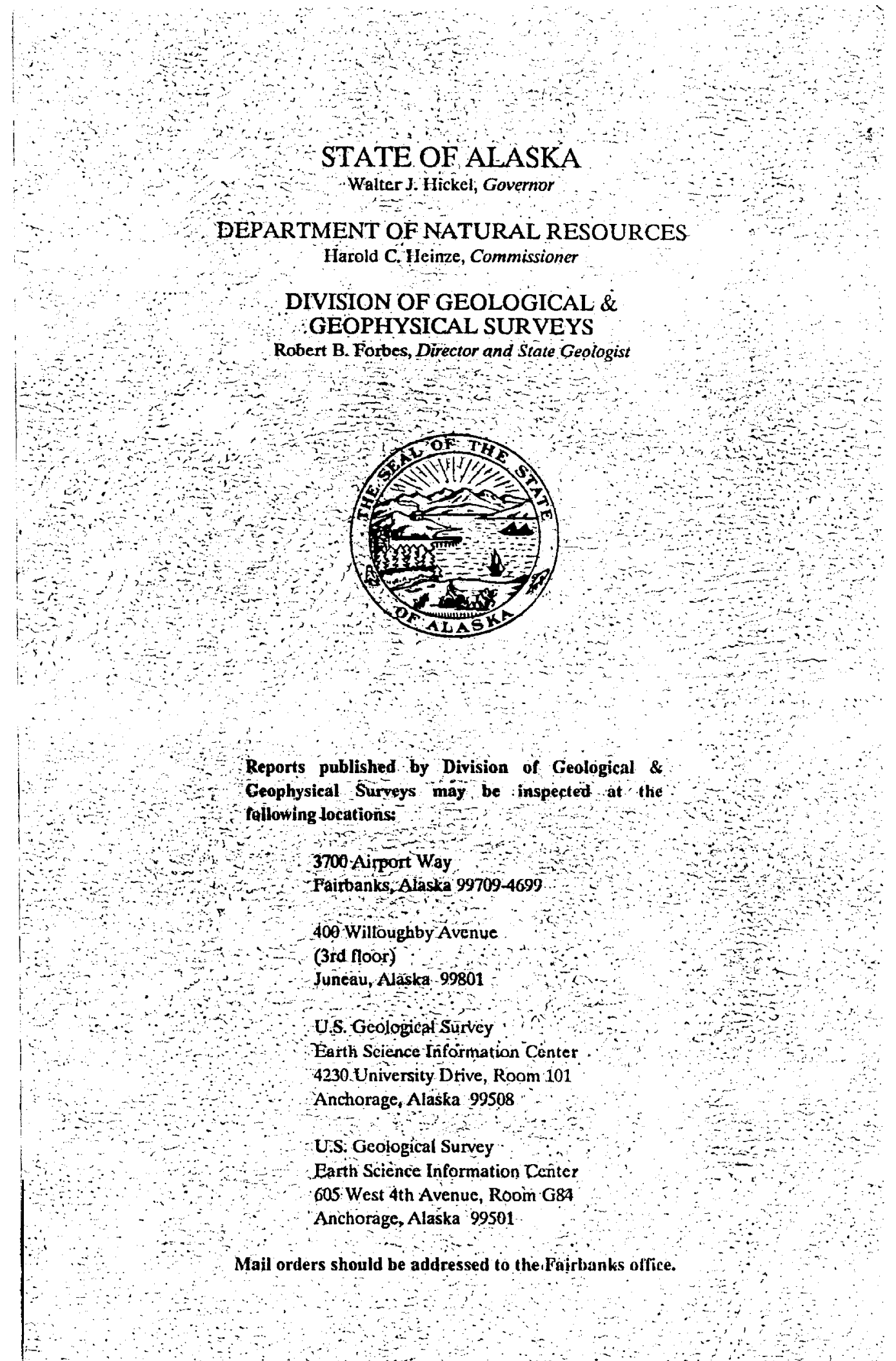

